\title{
Review of the Neotropical genus Prasmodon (Hymenoptera, Braconidae, Microgastrinae), with emphasis on species from Area de Conservación Guanacaste, northwestern Costa Rica
}

Jose L. Fernandez-Triana ${ }^{1,2, \dagger}$, James B. Whitfield ${ }^{3, \ddagger}$, M. Alex Smith ${ }^{1, \S}$, Yves Braet ${ }^{4, \S}$, Winnie Hallwachs ${ }^{5,1}$, Daniel H. Janzen ${ }^{5, \pi}$

I Department of Integrative Biology and the Biodiversity Institute of Ontario, University of Guelph, Guelph, ON N1G 2W1 Canada 2 Canadian National Collection of Insects, 960 Carling Ave., Ottawa, ON K1A OC6 Canada 3 Department of Entomology, University of Illinois, Urbana, IL 61801 USA 4 Unité d'Entomologie fonctionnelle et évolutive, Gembloux Agro-Bio Tech, Université de Liège, B-1030 Gembloux, Belgique; and Département d'entomologie, IRSNB, Rue Vautier 29, 1000 Bruxelles, Belgique 5 Department of Biology, University of Pennsylvania, Philadelphia, PA 19104-6018 USA

† http://zoobank.org/4469D91F-BBC1-4CBF-8263-EBFE2A95E4BF

¥ttp://zoobank.org/7A98AB5F-552D-4437-8F5D-C593CA713506

§ http://zoobank.org/E46EE6EB-E096-4FCD-BF5A-F91D4A8294EE

| http://zoobank.org/C1A1006D-C170-430D-9D9F-27AB0878DDDD

I http://zoobank.org/B2F86601-DF32-469C-942F-11116282C467

\# http://zoobank.org/4491369A-CFA6-4614-AC09-1137CCD06F9A

Corresponding author: Jose Fernandez-Triana (jftriana@uoguelph.ca)

Academic editor: G. Broad| Received 4 December 2013 | Accepted 14 March2014 | Published 28 March 2014

http://zoobank.org/6F9DA9D1-5000-45DB-ABOE-5212EF158781

Citation: Fernández-Triana JL, Whitfield JB, Smith MA, Braet Y, Hallwachs W, Janzen DH (2014) Review of the Neotropical genus Prasmodon (Hymenoptera, Braconidae, Microgastrinae), with emphasis on species from Area de Conservación Guanacaste, northwestern Costa Rica. Journal of Hymenoptera Research 37: 1-52. doi: $10.3897 /$ JHR.37.6748

\begin{abstract}
The Microgastrinae genus Prasmodon is revised and the following 16 new species are described: erenadupontae and verhoogdenokus (authored by Braet and Fernández-Triana), and almasolisae, aureus, bobpoolei, bobrobbinsi, dondavisi, johnbrowni, masoni, mikepoguei, nixoni, paulgoldsteini, scottmilleri, silvatlanticus, subfuscus, and tijucaensis (authored by Fernández-Triana and Whitfield). The greatest species richness is found in the Amazon basin, but the genus extends throughout the rain forests of Central and South America. Leaf-rolling and webbing caterpillars of Crambidae and Elachistidae are the only known hosts for these parasitoids.
\end{abstract}

Copyright Jose Fernandez-Triana et al. This is an open access article distributed under the terms of the Creative Commons Attribution License (CC BY 4.0), which permits unrestricted use, distribution, and reproduction in any medium, provided the original author and source are credited. 


\section{Keywords}

Microgastrinae, Prasmodon, parasitoid biology, Neotropics, Area de Conservación Guanacaste, Costa Rica, species descriptions, caterpillars

\section{Introduction}

The subfamily Microgastrinae (Hymenoptera: Braconidae) is a species-rich and taxonomically difficult lineage of parasitoid wasps that attack caterpillars (Lepidoptera), and also one of the main taxa used in the biological control of caterpillar pests in forestry and agriculture (Whitfield 1995, 1997). Close to 2,500 species have been described worldwide, although the total species richness may be as high as 40,000+ (Rodriguez et al. 2013).

The last two decades have seen an increase in the study of microgastrine wasps in the Neotropics - as a result of the publication of a key to New World genera (Whitfield 1997) and numerous biodiversity inventories carried out in the Neotropics. Among the sites more comprehensively studied, Area de Conservación Guanacaste (ACG), in northwestern Costa Rica, is an important and iconic effort to inventory all species of caterpillars, their food plants, and their parasitoids (Janzen et al. 2009). The fauna of ACG parasitoids has been the focus of many taxonomic revisions since the 1980's, and those of microgastrines in the past decade (Deans et al. 2003, Janzen et al. 2003, Janzen and Hallwachs 2011, Valerio et al. 2005, 2009, Grinter et al. 2009, Whitfield et al. 2012, Smith et al. 2007, 2008, Fernández-Triana et al. 2013, Fernández-Triana et al. 2014).

This paper, a continuation of those studies, deals with Prasmodon. This genus was described by Nixon (1965) as monotypic, based on a striking species from Peru, Prasmodon eminens Nixon. Forty years later, a second species, Prasmodon zlotnicki Valerio \& Rodriguez, was discovered and described from ACG (Valerio et al. 2005). Our study has found many additional undescribed Neotropical species in ACG and South America. Here we describe 16 of them, along with new host information when available. There are more species yet to describe, but we do not have sufficient specimens for that task at present.

Since Nixon's original description of Prasmodon, little progress has been made in determining its relationships with other microgastrine genera. Mason (1981) suggested a "Prasmodon group" based on his intuitive view of their phylogeny, without clarifying which genera might be included. Based on characters he mentions in his reclassification, however, it appears that the genera Clarkinella Mason, 1981 and Beyarslania Koçak \& Kemal, 2009 (the latter a recent replacement name for the preoccupied name Xenogaster Mason) might be candidates for inclusion in this group, possibly along with the more recently described genus Neoclarkinella Rema \& Narendran, 1996. Morphological study of many specimens of Pseudapanteles Ashmead, 1898 indicates some possibility of a close relationship with Prasmodon as well (although Mason treated it as a distinct lineage). Early molecular analyses with three genes (Mardulyn and Whitfield 1999; Whitfield et al. 2002) were inconclusive in resolving this possible relationship, but occasionally showed some possible 
relationship of Prasmodon to Pseudapanteles; when morphological data were added to the mix (Whitfield et al. 2002), a closer relationship with Clarkinella and Beyarslania (the latter named as Xenogaster in that paper) is suggested, but with low support. A more recent phylogenetic study incorporating seven genes and comparative morphology (Banks and Whitfield 2006) did not sample Clarkinella and Beyarslania, but again found Prasmodon to be related to Pseudapanteles with reasonably strong support. The matter still requires the analysis of both multiple genes and broader taxon sampling.

\section{Methods}

Most of the specimens used in this study came from ACG. They were reared (usually, see http://janzen.sas.upenn.edu for details for any particular specimen) or Malaisetrapped, and host caterpillars and other ecological information are considered along with DNA barcodes (COI gene) when available (Fig. 105). Additionally, a collection of Prasmodon specimens from French Guiana was available (personal collection of YB, collected during several field expeditions and also obtained through collaborations with various people living there). We also studied the South American specimens of Prasmodon in the Canadian National Collection of Insects (CNC) in Ottawa, Canada. The non-ACG material did not have associated host or molecular data, with the exception of a few, mostly short DNA barcodes (usually $160 \mathrm{bp}$ ) from some CNC specimens. Altogether 140+ specimens were studied.

The specimens of the new species are deposited in the CNC, Natural History Museum, London, United Kingdom (BMNH), the Illinois Natural History Survey, Champaign, Illinois, United States (INHS), the Instituto Nacional de Biodiversidad, Santo Domingo, Costa Rica (INBio), the National Museum of Natural History, the Smithsonian Institution, Washington DC, United States (NMNH), Naturalis Biodiversity Center Leiden, the Netherlands, (RMNH), Institut Royal des Sciences Naturelles de Belgique Brussels, Belgium (IRSNB), and Museum National d'Histoire Naturelle, Paris, France (MNHN).

Morphological terms and measurements of structures are mostly as used by Mason (1981), Huber and Sharkey (1993), Whitfield (1997), Karlsson and Ronquist (2012), and Fernández-Triana et al. (2014). Non-morphological characters are also provided in the key whenever available (e.g., geographical distribution, hosts). Those features are included in brackets at the end of the corresponding couplet and are intended as supplementary information to aid identification.

The new species descriptions are based on the holotype female, with other specimens studied (when available) for intraspecific variation.

Lucid 3.5.4 (http://www.lucidcentral.com/) software was used to generate automatic descriptions of the species and to prepare Lucid identification keys. A dataset of 22 characters and 90 character-states was used to provide uniform descriptions for all species treated. The description format includes one sentence per character, with the character mentioned first and the character-state following after a colon, e.g., "Scape 
color: entirely yellow". Whenever a species scored more than one character-state, the description included all of the pertaining character-states separated by "or", e.g., "Scape color: entirely yellow or partially brown to black". Whenever a character-state was coded as uncertain due to poor condition of the specimen(s), the description includes the details of the character-state as best assessed, followed by a question mark, e.g., "Scape color: entirely yellow (?)". Sometimes a character could not be coded due to missing body parts in the available specimens; in such instances the feature was left out of the description for that particular species.

In most cases we used a simplified convention to code color, considering it as either pale (white, light yellow, orange-yellow, light brown-yellow) or dark (dark brown, black). For details on the exact color patterns on the body, we provide extensive illustrations for every species.

Photos were taken with a Keyence VHX-1000 Digital Microscope, using a lens with a range of 13-130x. Multiple images through the focal plane were taken of a structure and these were combined to produce a single in-focus image, using the software associated with the Keyence System.

A map with the distribution of all species was generated using SimpleMappr (Shorthouse 2010).

DNA barcodes for all ACG inventory Microgastrinae were obtained using DNA extracts prepared from single legs using a glass fibre protocol (Ivanova et al. 2006). Extracts were re-suspended in $30 \mu \mathrm{l} \mathrm{of} \mathrm{dH2O}$, and a 658-bp region near the $5^{\prime}$ terminus of the COI gene was amplified using standard primers (LepF1-LepR1) following established protocols (Smith et al. 2006, 2007, 2008). If the initial 658 bp amplification was not successful composite sequences were generated using internal primers. Primer information for individual sequences can be retrieved from the Barcode of Life Data System (BOLD) (Ratnasingham and Hebert 2007), but primers are as detailed in Smith et al. (2008). DNA barcoding data and related information for all specimens studied in this paper can be accessed at: dx.doi.org/10.5883/DS-ASPRAS.

In the taxonomic treatment of species, "Specimens Examined" presents the specimen's information in the following format: "Number of females/males examined, acronym of the storing institution between parenthesis, COUNTRY: State/Province, Region, city, other locality details, latitude/longitude coordinates (in decimal degrees), collection date, collector name, biological information on host (starting with "ex"), ACG database codes (in the format "yy-SRNP-xxxxxx" for the host caterpillar or parasitoid lot reared from it, or "DHJPARxxxxxxx" for an individual parasitoid specimen).

\section{Results}

We describe here 16 new species from seven countries (Brazil, Colombia, Costa Rica, Ecuador, French Guiana, Peru and Suriname); increasing the number of Prasmodon 
species from two to 18 . There are at least three additional species (one each from Brazil, Costa Rica and Ecuador) in the CNC collection, only represented by 1-2 males each, with no molecular or biological data associated. Those species will remain undescribed until more specimens, especially females, become available. Males that cannot be associated with females have usually proven to be almost useless in Microgastrinae taxonomy (e.g., Austin 1990; Whitfield 1997).

The distribution of species by country and by major bioregion shows some interesting patterns (Fig. 104). We found 10 species of Prasmodon in Costa Rica, out of roughly 140 rearings and 8 Malaise-trapped specimens from ACG. Seven species were recorded from Brazil, out of some 30 Malaise-trapped specimens. Ecuador (6 specimens), French Guiana (20 specimens), and Peru (3 specimens) had three species each. Colombia (3 specimens) and Suriname (1 specimen) had one species each.

Altogether, Central America (only known specimens are from ACG) had 10 of the total species found (55.6\%), while South America had nine species (50 \%). Prasmodon eminens was the only species shared between Central and South America (Fig. 104), but even that might be an artifact because that species is thought to actually comprise a complex of morphologically cryptic species (e.g. Valerio et al. 2005). The South American fauna has been much more poorly sampled than has been that of ACG, and many fewer South American specimens were available for us to study; it is very likely that more collecting will reveal a substantial number of new species.

As for major bioregions, ACG had 10 species, sharing one of them with the Amazonia bioregion (for simplicity, we consider here ACG as one region altogether, even though it is a mosaic of ecosystems, just as the other so-called "bioregions"). Amazonia had six species, one shared with ACG, and three shared with the Guyana lowland rainforests (which has no endemic species on its own). The Atlantic Forest of Brazil had three species, all endemic to that region (Fig. 104).

The ACG Prasmodon specimens have been collected in relatively low-elevation areas $(95-980 \mathrm{~m})$, in intact rain forest or secondary vegetation where there used to be intact rain forest 0-100 years earlier, except for Prasmodon paulgoldsteini, which has been found (6 Malaise-trapped specimens) only in ACG dry forest at about $300 \mathrm{~m}$ elevation. Based on the data currently available, Prasmodon is largely a genus of Neotropical rain forests. Although the most species-rich area at present is Central America (ACG), we speculate that further collecting will reveal the highest diversity to be actually found in South America.

In a first analysis of the biology of the genus, Valerio et al. (2005) reported that species of Prasmodon in ACG are solitary parasitoids of leaf-rolling or leaf-webbing Spilomelinae (Crambidae), feeding on shrubs, treelets and tree saplings across a wide range of plant families (Cyathaceae, Araliaceae, Euphorbiaceae, Fabaceae). Successive sampling and improved taxonomy of the hosts in ACG has revealed that the only hosts are Crambidae and Elachistidae (see host records associated with each species below). Hosts are only known for the ACG specimens. 


\section{Diagnosis of the genus Prasmodon}

This is one of the most recognizable genera of Microgastrinae (Mason 1981, Whitfield 1997, Valerio et al. 2005). The body size (3.3-5.8 mm, with most species $4.0 \mathrm{~mm}$ or more) and the fore wing length $(3.8-6.2 \mathrm{~mm}$, with most species $4.2 \mathrm{~mm}$ or more) are among the largest within microgastrine wasps. The body coloration is mostly yellow-orange, with the wings usually infumate (e.g. Figs $1,2,7,8,13,14)$ ). The notauli are deeply impressed (Figs $5,11,17,22,27,32,38,44,50,56,62,68,73,80,86,92,94,95)$. The fore wing has a closed areolet (second submarginal cell), and the vannal lobe in the hind wing is reduced (e.g., Figs 17, 22, 63, 75, 95). The propodeum has a clearly defined transverse carina crossing the median longitudinal carina (e.g. Figs 5, 17, 39, 45). The hypopygium is inflexible (e.g., Figs 43, 55, 70). The combination of body size and color, notauli, transverse carina on propodeum, and inflexible hypopygium are particularly useful to distinguish Prasmodon.

\section{Key to species of Prasmodon}

[Throughout the key the acronyms T1, T2, etc., are used for morphological terms mediotergite 1, 2, etc.; and F refers to flagellomere number, e.g. F12]

1 Mesosoma and metasoma entirely yellow-orange dorsally (Figs 5, 6, 11, 12, $17,18,27,28,32,34,38,39,44,45,56,58,92,93)$, except for very small black spot on axillar complex (as in Fig. 5) .2

- $\quad$ Mesosoma and/or metasoma with some brown coloration dorsally (Figs 23, $50,51,63,69,75,80,81,86,87,94,95)$, in addition to very small black spot on axillar complex

2(1) Apical flagellomeres yellow or yellowish-white, contrasting with rest brown to black (Figs 3, 13, 30, 54, 90) .............................................................. 3

- $\quad$ All flagellomeres brown to black (as in Figs 11, 12, 24, 40) ......................

3(2) Antenna mostly dark brown to black; with small, apical area (F15-16, and occasionally apical half of F14) which is yellow-brown, lighter in colour than remaining flagellomeres (Figs 3, 13)...

- $\quad$ Antenna with yellow area much larger (at least including F12-15, and usually apical half of F11 and basal half of F16), much lighter compared to remaining flagellomeres (Figs 30, 54)

4(3) Metatarsus almost entirely yellow-white (Fig. 13); posterior $0.1-0.2$ of mesotibia dark brown to black; body length $4.2-4.3 \mathrm{~mm}(\mathrm{X}=4.3 \mathrm{~mm})$, rarely 4.5 $\mathrm{mm}$; fore wing length $4.5-4.6 \mathrm{~mm}(\mathrm{X}=4.6 \mathrm{~mm})$, rarely $4.8 \mathrm{~mm}$; T1 $5.6 \times$ as long as width at posterior margin [Hosts: Elachistidae, mostly Anteotricha feeding on Bignoniaceae]

Prasmodon bobpoolei Fernández-Triana \& Whitfield, sp. n.

- $\quad$ Metatarsus dark brown to black (except for anterior 0.7 of first metatarsomere) (Fig. 1); mesotibia entirely yellow; body length 4.5-4.9 mm (X=4.8 
$\mathrm{mm})$; fore wing length $5.0-5.3 \mathrm{~mm}(\mathrm{X}=5.1 \mathrm{~mm})$; T1 $4.0 \times$ as long as width at posterior margin [Hosts: Elachistidae, mostly Antaeotricha feeding on Meliaceae] ...... Prasmodon almasolisae Fernández-Triana \& Whitfield, sp. n.

5(3) Fore wing with a pale area centrally (Figs 30, 33) which gives the wing a banded appearance; anterior $0.4-0.5$ of vein $3 \mathrm{M}$ (and usually part or all of veins $2 \mathrm{M}$ and $\mathrm{r}-\mathrm{m}$ ) yellow-orange, contrasting with all other veins which are dark brown (Fig. 33); scutoscutellar sulcus with 4 impressions (Fig. 32); T1 distinctly narrowing at around 0.5 its length, with both anterior and posterior margins clearly wider than its median width (Fig. 34)

Prasmodon erenadupontae Braet \& Fernández-Triana, sp. n.

- $\quad$ Fore wing either mostly hyaline, or entirely infumate (except for small hyaline area near veins $(\mathrm{RS}+\mathrm{M}) \mathrm{b}$ and $2 \mathrm{M})$; all veins dark brown (Figs 53, 89); scutoscutellar sulcus usually with more than 5 impressions (Figs 56, 92); T1 distinctly narrowing posteriorly, width at posterior margin clearly less than width at anterior margin and median width (Figs 58, 89, 93) ................6

6(5) Hypostomal carina not raised (e.g. Figs 16, 57); hind wing subbasal cell mostly setose (Fig. 44); T1 less than $5.0 \times$ as long as width at posterior margin; T2 $5.0 \times$ as wide at posterior margin as median length

Prasmodon nixoni Fernández-Triana \& Whitfield, sp. n.

- Hypostomal carina highly raised (Figs 9, 25, 31); hind wing subbasal cell mostly without setae (Fig. 39); T1 $6.0 \times$ as long as width at posterior margin; T2 less than $3.0 \times$ as wide at posterior margin as median length

Prasmodon verboogdenokus Braet \& Fernández-Triana, sp. n.

7(2) Ovipositor sheaths relatively short, $0.3 \times$ as long as metatibia length (Fig. 43); all legs entirely yellow (Fig. 40)

Prasmodon masoni Fernández-Triana \& Whitfield, sp. n. Ovipositor much longer, 0.5-0.6 $\times$ as long as metatibia length (Figs 7, 24, $35)$; at least posterior $0.1-0.2$ of metatibia and most of metatarsus (and sometimes parts of middle leg) dark brown to black (Figs 7, 26, 35, 37)....8

8(7) Scape entirely yellow (Fig. 9); fore wing with most veins golden yellow, pterostigma mostly yellow, with central part brown (Figs 8, 10) [Ecuador]

Prasmodon aureus Fernández-Triana \& Whitfield, sp. n. Scape partially dark brown to black (Figs 25, 35, 38); fore wing with most veins and pterostigma entirely dark brown (Figs 24, 36) [Costa Rica, ACG]

9(8) Posterior $0.1-0.2$ of mesotibia, apical segment of mesotarsus, posterior $0.2-0.3$ of metatibia, and metatibia spurs, dark brown to black (Figs 35, 37); scutoscutellar sulcus with 5-6 impressions (Fig. 38); hind wing with subbasal cell mostly without setae (Fig. 39); T1 evenly narrowing towards posterior margin, its length $6.3 \times$ as width at posterior margin (Fig. 39) [Hosts: Crambidae, Asturodes fimbriauralis DHJ02, Eulepte alialis, Piletosoma thialis, Phostria Janzen05, feeding on many plant families].. Prasmodon johnbrowni Fernández-Triana $\&$ Whitfield, sp. n.

- $\quad$ Mesotibia, mesotarsus, and metatibia spurs entirely yellow-orange (Figs 24, 26); posterior $0.1-0.2$ of metatibia dark brown to black; scutoscutellar sulcus 
with 4 impressions (Fig. 27); hind wing with subbasal cell mostly setose (although it may be a small area without setae near lower margin of wing, as in Fig. 28); T1 more or less parallel-side until half its length, then slightly narrowing towards posterior margin (Figs 27, 28), its length 3.3-3.7 $\times$ as width at posterior margin [Hosts: Crambidae, Prenesta feeding on Apocynaceae: Prenesta Janzen196, Prenesta Janzen 195, Prenesta scyllalisDHJ03]

Prasmodon dondavisi Fernández-Triana \& Whitfield, sp. n.

10(1) Only metasoma with some brown coloration on mediotergites; mesosoma completely yellow-orange (Figs 22, 23, 62, 63, 68, 69, 73, 75, 95)

- Both mesosoma and metasoma with brown to black areas (Figs 50, 51, 80, $81,86,87,94)$.

11(10) Metasoma almost completely yellow-orange (Fig. 95), with only small brown areas centrally on mediotergites 4-7 (usually only on mediotergires 5-6); larger species, body length 5.0-5.3 $\mathrm{mm}$ and fore wing length 5.2-5.6 mm...

Prasmodon eminens Nixon, 1965

- Metasoma with brown to black areas covering most of mediotergites 4-7, and also part of mediotergites 3 and 8 (Figs 23, 63, 69, 75); smaller species, body length 3.8-4.0 $\mathrm{mm}$ and fore wing length $4.0-4.6 \mathrm{~mm}$.....

12(11) All coxae, pronotum, mesopleuron, metapleuron, and propodeum white to very light yellow, contrasting with rest of mesosoma yellow to orange-yellow (Figs 64, 68, 69); T1 $3.7 \times$ as long as width at posterior margin. [Hosts: Crambidae, Omiodes, Triuncidia, feeding on many plant families]

Prasmodon scottmilleri Fernández-Triana \& Whitfield, sp. n. Metacoxa, pronotum, most of mesopleuron, metapleuron and at least anterior 0.5 of propodeum yellow-orange, same color than most of mesosoma (Figs 19, 22, 23, 59, 61-63, 70, 72, 75); T1 5.0-6.0 $\times$ as long as width at posterior margin 13

13(12) Humeral complex, part of axillar complex, and scutoscutellar sulcus dark brown to black; notauli very deeply impressed and with darker coloration than rest of anteromesoscutum (Fig. 73); hind wing subbasal cell mostly without setae (Figs 71, 75) [Brazil]

Prasmodon silvatlanticus Fernández-Triana \& Whitfield, sp. n.

- Humeral complex, axillar complex, and scutoscutellar sulcus orange-yellow, same colour than rest of mesosoma (Figs 22, 62); notauli not as strongly impressed as above, and same coloration than rest of anteromesoscutum; hind wing subbasal cell usually mostly setose (as in Fig. 63) [Costa Rica, ACG] ............. 14

14(13) Propodeum with median longitudinal and transverse carinae clearly marked, without additional carination pattern (Fig. 23); T3-T7 mostly brown (Figs 19, 23) [Host: Crambidae, Palpita jairusalis DHJ01 feeding on Apocynaceae]........... Prasmodon bobrobbinsi Fernández-Triana \& Whitfield, sp. n.

- $\quad$ Propodeum with irregular carinae in addition to median longitudinal and transverse carinae (Fig. 63); T3 yellow-orange; T4-T7 mostly orange with brown coloration restricted to central part of tergites (Fig. 63) [Host: un- 
known, Malaise-trapped]

Prasmodon paulgoldsteini Fernández-Triana \& Whitfield, sp. n.

15(10) Mesosoma with extensive brown coloration: anteromesoscutum mid-frontal and lateral areas, lateral face of scutellum, axillar complex, and metascutellum (Figs 76, 77, 80, 81); T1 with posterior $0.2-0.3$ brown (Figs 77, 81); metacoxa with small brown spot on posterior 0.1 (Fig. 76); metatrochanter and metatrochantellus brown

Prasmodon subfuscus Fernández-Triana \& Whitfield, sp. n.

- Mesosoma at most with anteromesoscutum mid-frontal and lateral areas brown (Figs 46, 50, 82, 86, 94); T1, metacoxa, metatrochanter and metatrochantellus fully yellow-white or yellow-orange (Figs 51, 82, 87, 94) 16

16(15) Metatibia and metatarsus entirely yellow (Figs 82, 85); wings hyaline, at most with very faint infumation on anterior 0.5 (Fig. 83); anteromesoscutum with only lateral areas brown, rest orange-yellow (Fig. 86) [Brazil]

Prasmodon tijucaensis Fernández-Triana \& Whitfield, sp. n.

- $\quad$ Posterior $0.1-0.2$ of metatibia and most of metatarsus dark brown to black (Figs 46, 49, 94); wings infumate (Fig. 47); anteromesoscutum usually with mid-frontal and lateral areas brown, rest orange-yellow (Fig. 50) [Costa Rica, ACG] ....17

17(16) Metasoma with only thin brown areas centrally on T4-T8 (Fig. 94); hind wing subbasal cell mostly without setae (Fig. 94) [Hosts: Crambidae, Ategumia, Desmia, Diacme, Herpetogramma, Phostria feeding on five plant families]

Prasmodon zlotnicki Valerio \& Rodriguez, 2005

- $\quad$ Metasoma with most of T3-T8 dark brown (Figs 46, 51); hind wing subbasal cell mostly setose (Figs 48, 50) [Hosts: Elachistidae feeding on three plant families] .... Prasmodon mikepoguei Fernández-Triana \& Whitfield, sp. n.

\section{Taxonomic treatment of species, in alphabetical order}

Prasmodon almasolisae Fernández-Triana $\&$ Whitfield, sp. n. http://zoobank.org/3C6F2705-DC55-4192-89E1-66FF9BC2F570 http://species-id.net/wiki/Prasmodon_almasolisae

Figs 1-6, 101

Type locality. COSTA RICA, ACG, Alajuela Province, Sector Rincon Rain Forest, Sendero Anonas, 405m, 10.90528, -85.27882.

Holotype. + in CNC. Specimen labels: 1. DHJPAR0038908. 2. Voucher: D.H.Janzen \& W.Hallwachs, DB: http://janzen.sas.upenn.edu, Area de Conservación Guanacaste, COSTA RICA, 10-SRNP-40859.

Paratypes. 2 क, 2 o (CNC, NMNH). COSTA RICA, ACG database codes: DHJPAR0038170, DHJPAR0038913, DHJPAR0040001, DHJPAR0040518.

Description. Female. Body length 4.9-5.0 mm, rarely 4.5-4.6 mm. Fore wing length 4.9-5.0 mm, 5.1-5.2 mm, rarely 5.3-5.4 mm. Body color: meso- and meta- 

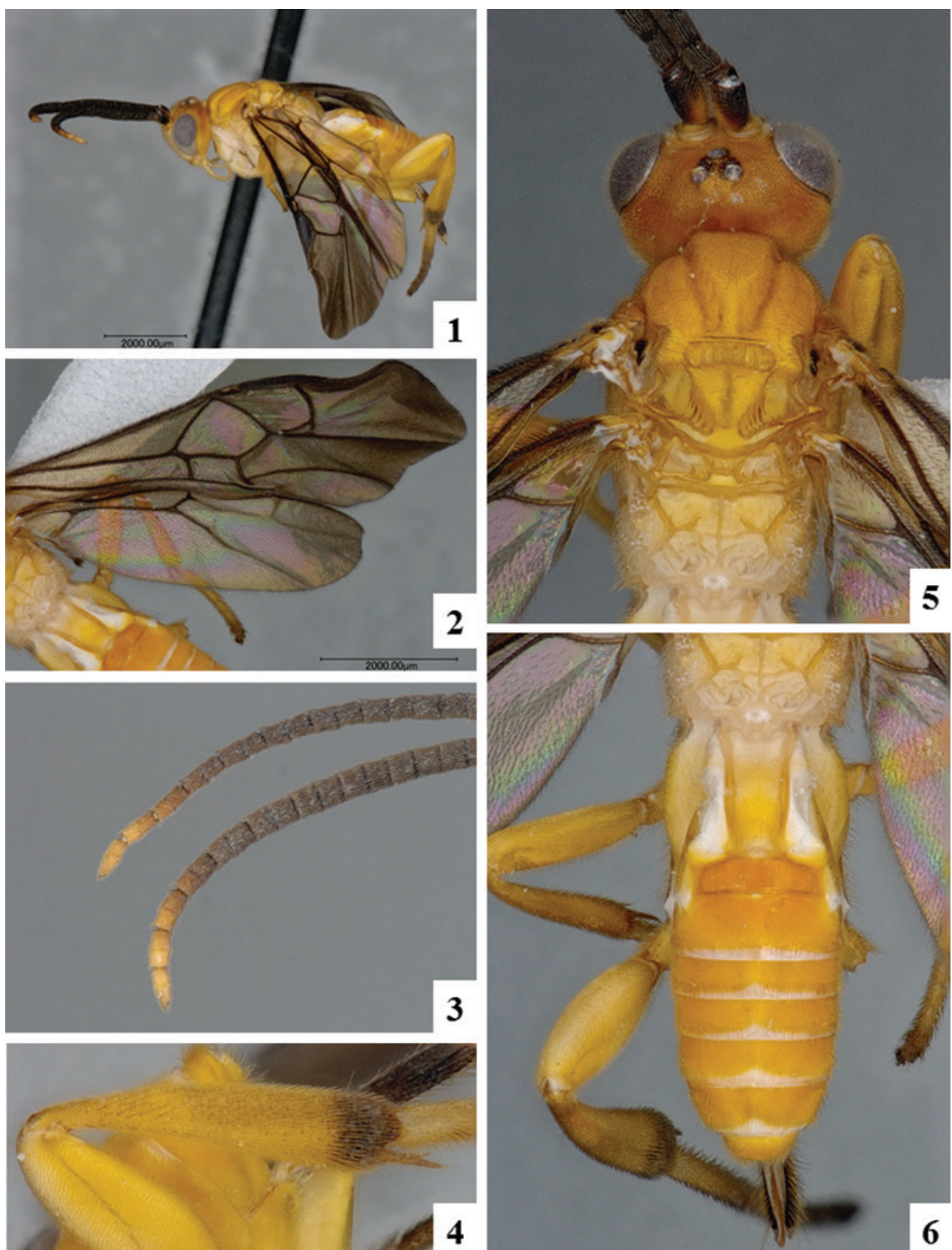

Figures I-6. Prasmodon almasolisae I Habitus 2 Fore wing 3 Antenna (partially) 4 Metatibia 5 Head and mesosoma, dorsal view $\mathbf{6}$ Propodeum and metasoma, dorsal view.

soma entirely yellow-orange (with the exception of a very small black spot on axillar complex) (Figs 5, 6). Scape color: partially dark brown to black (Fig. 5). Flagellomeres color: most flagellomeres brown to black, except for small apical area (F15-16, and occasionally apical half of F14) which is yellow-brown (Fig. 3). Tegula and humeral 
complex color: tegula pale, humeral complex partially dark/partialy pale. Mesotibia color: entirely yellow. Metatibia color: posterior 0.1-0.3 dark brown to black (Fig. 4). Metatibia spurs color: yellow-orange. Metatarsus color: dark brown to black (except for anterior 0.7 or less of first metatarsomere) (Fig. 1). Fore wing color pattern: hyaline. Fore wing veins color: all veins dark brown (Fig. 2). Pterostigma color: entirely dark brown. Hypostomal carina: not raised. Scutoscutellar sulcus: with 6 impressions (Fig. 5). Areolet height $\div$ vein $\mathrm{r}$ length (fore wing): $0.2 \times$. Hind wing subbasal cell: mostly without setae (Fig. 5). Hind tarsal claws: with pectination uniform, teeth thick and relatively evenly spaced. Shape of mediotergite 1: distinctly narrowing posteriorly, width at posterior margin clearly less than width at anterior margin and median width (Fig. 6). Mediotergite 1 length $\div$ width at posterior margin 3.6-4.0 $\times$. Mediotergite 2 width at posterior margin $\div$ length: $3.1-3.5 \times$. Ovipositor sheaths length: $0.6 \times$ as long as metatibia, rarely $0.5 \times$ as long as metatibia.

Male. As female but with flagellomeres fully dark brown to black.

Molecular data. Sequences in BOLD: 11, barcode compliant sequences: 11 (Fig. 105).

Biology and ecology. Hosts: Elachistidae, Antaeotricha radicalis, Antaeotricha ribbei, Antaeotricha Janzen07, Antaeotricha thapsinopa, elachJanzen01 Janzen131, Gonionota Janzen116, Stenoma Janzen129.

Distribution. Costa Rica, ACG rain forest.

Etymology. This species is named in honour of Alma Solis of the SEL/USDA laboratory in the National Museum of Natural History, Smithsonian Institution, Washington, D.C, in recognition of her decades of taxonomic knowledge and support contributing to understanding the species-level and higher taxonomy of the Crambidae, Pyralidae and Thyrididae of ACG, INBio and Costa Rica, as well as other parts of the world.

\section{Prasmodon aureus Fernández-Triana $\&$ Whitfield, sp. n. http://zoobank.org/306A4FAB-DCD8-42C0-981F-C6B8D97EDC64 http://species-id.net/wiki/Prasmodon_aureus}

Figs $7-12$

Type locality. ECUADOR, Napo, Limoncocha, $250 \mathrm{~m}$.

Holotype. + in CNC. Specimen labels: 1. ECUADOR, Napo, Limoncocha, 250m, 15-28.VI.1976, S. \& J. Peck. 2. DNA Voucher, CNCHYM 01955.

Description. Female. Body length 5.5-5.6 mm. Fore wing length 5.9-6.0 mm. Body color: meso- and metasoma entirely yellow-orange (with the exception of a very small black spot on axillar complex) (Figs 7, 11, 12). Scape color: entirely yellow (Fig. 11). Flagellomeres color: all flagellomeres brown to black (Figs 11, 12). Tegula and humeral complex color: both pale. Mesotibia color: entirely yellow. Metatibia color: posterior 0.1-0.3 dark brown to black. Metatibia spurs color: yellow-orange. Metatarsus color: dark brown to black (except for anterior 0.7 or less of first metatarsomere). Fore wing color pattern: uniformly and entirely infumate (except for small hyaline area 

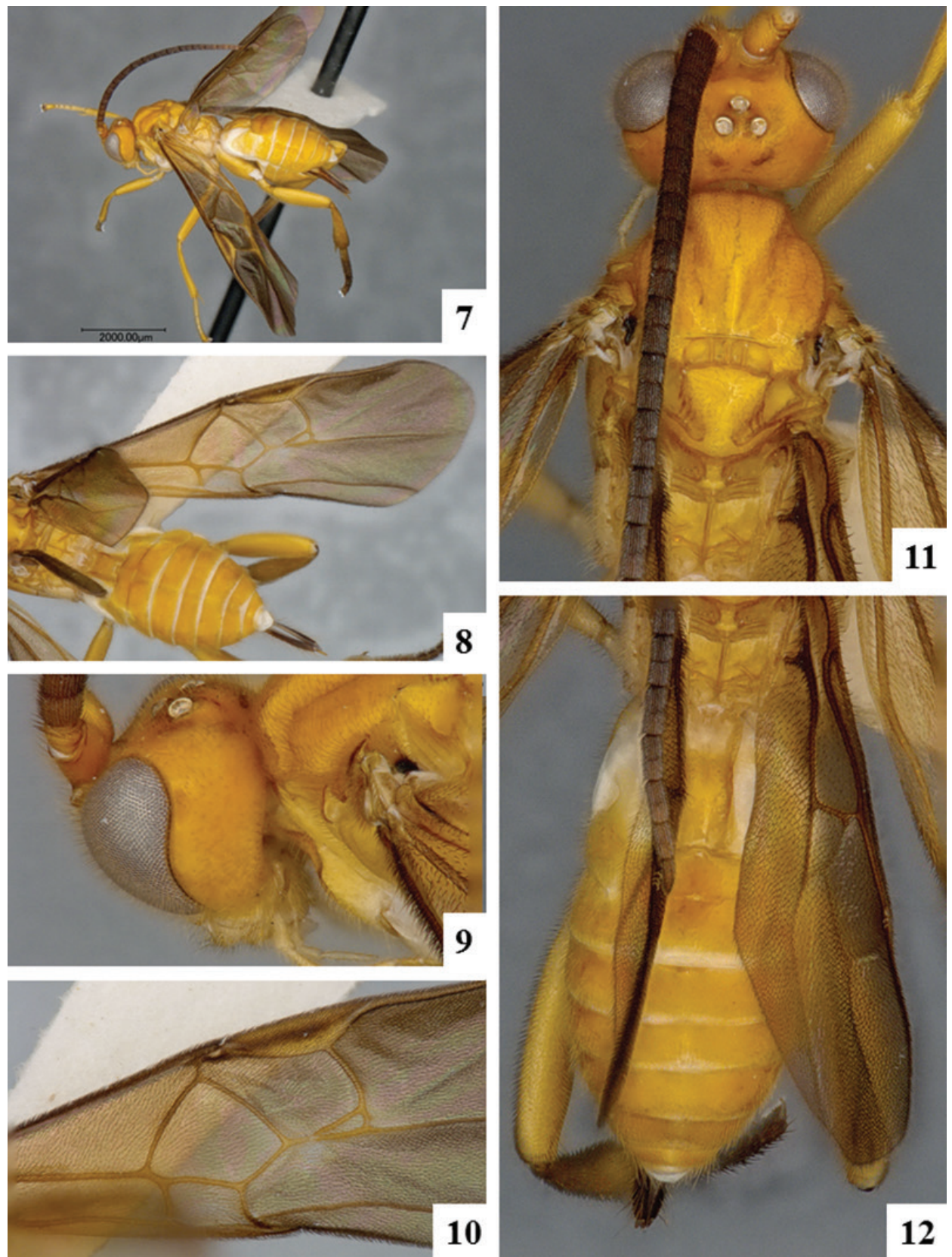

Figures 7-12. Prasmodon aureus. 7 Habitus 8 Fore wing 9 Head and mesosoma (partially), lateral view 10 Detail of fore wing I I Head and mesosoma, dorsal view $\mathbf{2}$ Propodeum and metasoma, dorsal view.

near veins $(\mathrm{RS}+\mathrm{M}) \mathrm{b}$ and $2 \mathrm{M})$. Fore wing veins color: most veins golden yellow (Figs 8, 10). Pterostigma color: mostly yellow, with central part brown (Fig. 10). Hypostomal carina: highly raised (Fig. 9). Scutoscutellar sulcus: with 4 impressions (Fig. 11). 
Areolet height $\div$ vein $r$ length (fore wing): $0.2 \times$. Hind wing subbasal cell: mostly with setae. Hind tarsal claws: with pectination uniform, teeth thick and relatively evenly spaced. Shape of mediotergite 1: distinctly narrowing posteriorly, width at posterior margin clearly less than width at anterior margin and median width. Mediotergite 1 length $\div$ width at posterior margin 6.1-6.5 $\times$. Mediotergite 2 width at posterior margin $\div$ length: $3.1-3.5 \times$. Ovipositor sheaths length: $0.5 \times$ as long as metatibia.

Male. Unknown.

Molecular data. Sequences in BOLD: 1, barcode compliant sequences: 0 .

Biology and ecology. Unknown.

Distribution. Ecuador, Napo.

Etymology. From Latin "aureus"= golden, referring to the yellow coloration of the fore wing veins, a unique feature among all known species of Prasmodon.

\section{Prasmodon bobpoolei Fernández-Triana \& Whitfield, sp. n. \\ http://zoobank.org/00CA5006-4B13-4D9C-A7A7-58A6841F7192 \\ http://species-id.net/wiki/Prasmodon_bobpoolei}

Figs 13-18, 99

Type locality. COSTA RICA, ACG, Alajuela, Province, Sector Rincon Rain Forest, Montanya Figueres, 460m, 10.88367, -85.29081.

Holotype. + in CNC. Specimen labels: 1. DHJPAR0045302. 2. Voucher: D.H.Janzen \& W.Hallwachs, DB: http://janzen.sas.upenn.edu, Area de Conservación Guanacaste, COSTA RICA, 11-SRNP-42719.

Paratype. 4 q, $2 \curvearrowright$ (CNC, NMNH). COSTA RICA, ACG database codes: DHJPAR0038211, DHJPAR0038246, DHJPAR0039997, DHJPAR0040003, DHJPAR0040005, DHJPAR0045165.

Description. Female. Body length 4.1-4.2 mm, 4.3-4.4 mm, rarely $4.5-4.6 \mathrm{~mm}$. Fore wing length $4.5-4.6 \mathrm{~mm}$, rarely $4.7-4.8 \mathrm{~mm}$. Body color: meso- and metasoma entirely yellow-orange (with the exception of a very small black spot on axillar complex). Scape color: partially dark brown to black (Figs 17, 18). Flagellomeres color: most flagellomeres brown to black, except for small apical area (F15-16, and occasionally apical half of F14) which is yellow-brown (Fig. 13). Tegula and humeral complex color: tegula pale, humeral complex partially dark/partialy pale. Mesotibia color: posterior $0.1-0.2$ dark brown to black. Metatibia color: posterior $0.1-0.3$ dark brown to black (Fig. 15). Metatibia spurs color: yellow-orange (Fig. 15). Metatarsus color: entirely yellow-white. Fore wing color pattern: uniformly and entirely infumate (except for small hyaline area near veins $(\mathrm{RS}+\mathrm{M}) \mathrm{b}$ and $2 \mathrm{M})$. Fore wing veins color: all veins dark brown (Fig. 14). Pterostigma color: entirely dark brown. Hypostomal carina: not raised (Fig. 16). Scutoscutellar sulcus: with 5 impressions (Fig. 17). Areolet height $\div$ vein $r$ length (fore wing): $0.2 \times$. Hind wing subbasal cell: mostly without setae. Hind tarsal claws: with pectination uniform, teeth thick and relatively evenly spaced. Shape of mediotergite 1: distinctly narrowing posteriorly, width at posterior margin clearly less than 

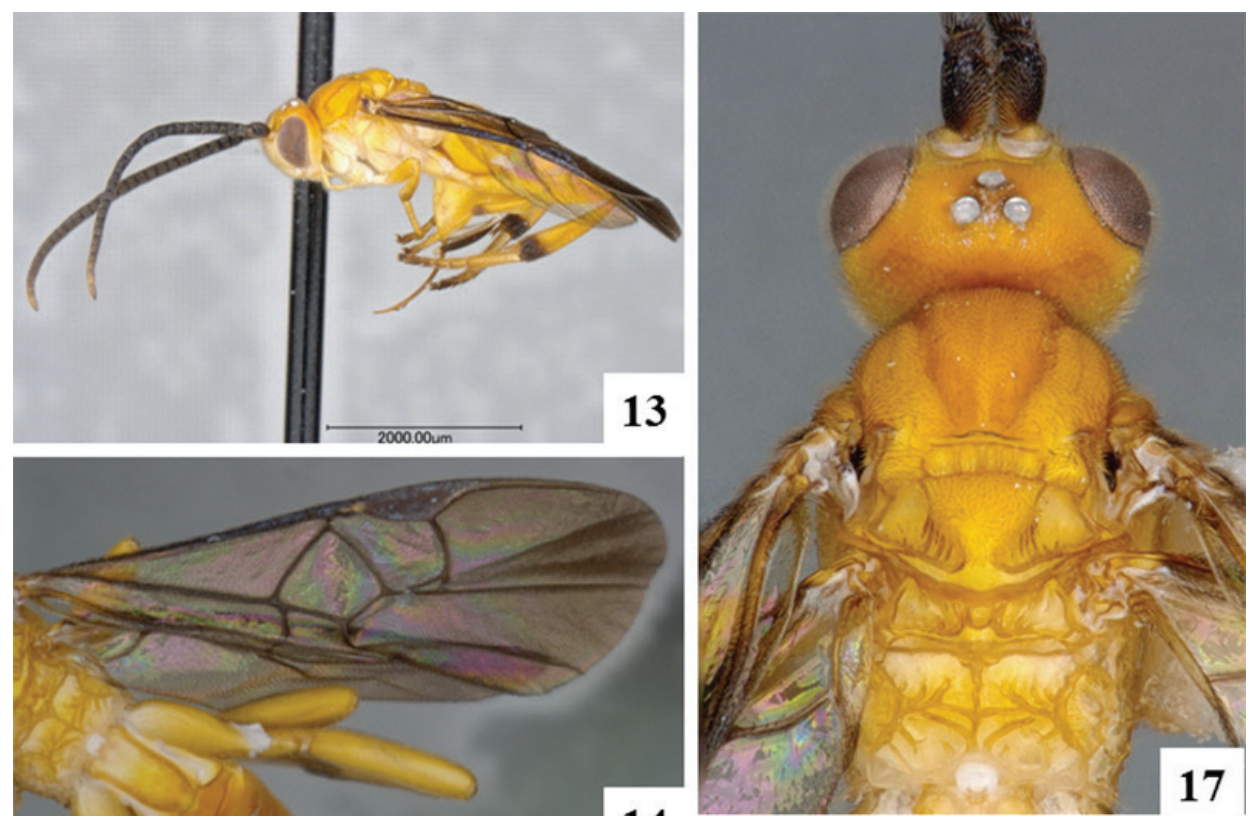

14
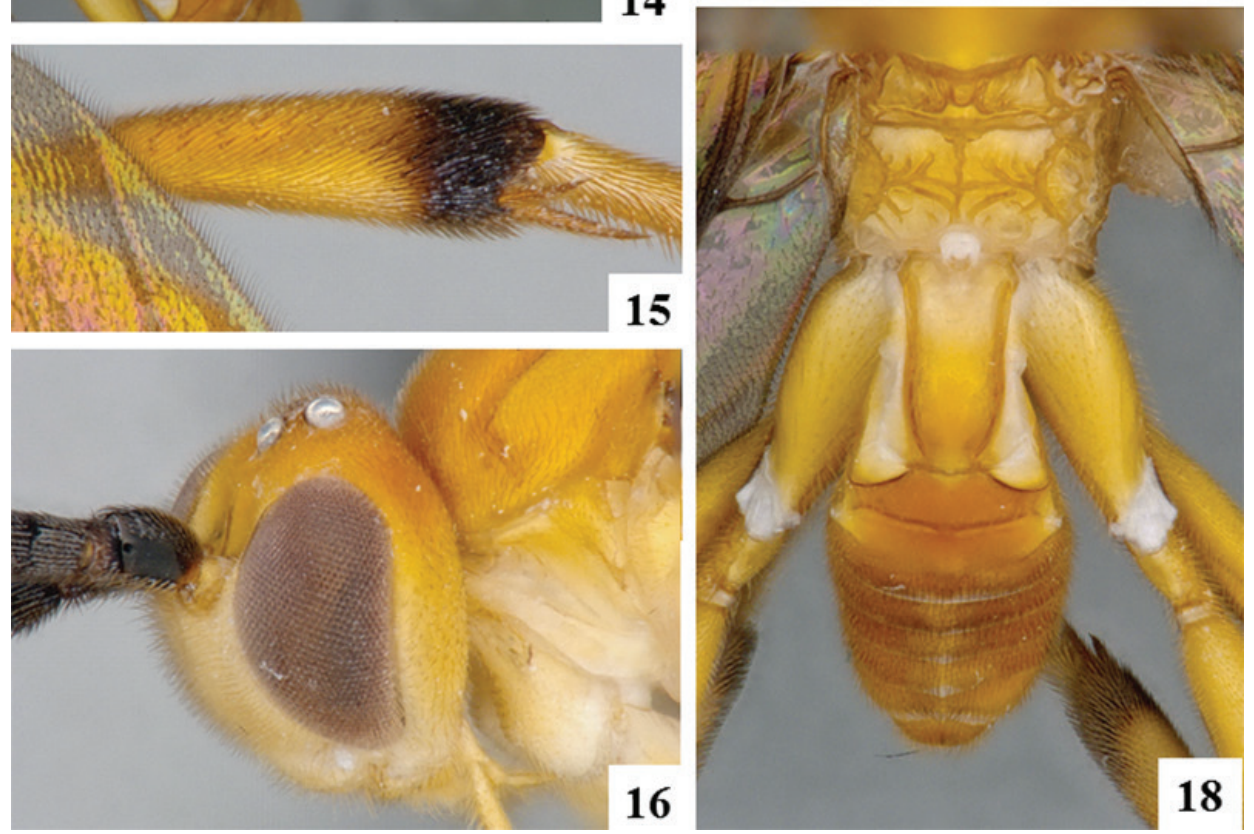

Figures 13-18. Prasmodon bobpoolei. 13 Habitus 14 Fore wing 15 Metatibia 16 Head and mesosoma (partially), lateral view $\mathbf{1 7}$ Head and mesosoma, dorsal view $\mathbf{1 8}$ Propodeum and metasoma, dorsal view.

width at anterior margin and median width (Fig. 18). Mediotergite 1 length $\div$ width at posterior margin 5.6-6.0 $\times$. Mediotergite 2 width at posterior margin $\div$ length: 2.6-3.0 $\times$. Ovipositor sheaths length: $0.6 \times$ as long as metatibia, rarely $0.5 \times$ as long as metatibia. 
Male. As female but with flagellomeres fully dark brown to black.

Molecular data. Sequences in BOLD: 15, barcode compliant sequences: 15 (Fig. 105).

Biology and ecology. Host: Elachistidae, Antaeotricha BioLep38, Antaeotricha Janzen110, Antaeotricha Janzen126, Gonioterma Janzen212, Stenoma Janzen 129.

Distribution. Costa Rica, ACG rain forest.

Etymology. This species is named in honour of Bob Poole of the National Museum of Natural History, Smithsonian Institution, Washington, D.C, in recognition of his decades of taxonomic knowledge and support contributing to understanding the species-level and higher taxonomy of the Noctuoidea and Geometroidea of ACG, INBio and Costa Rica, as well as other parts of the world.

\section{Prasmodon bobrobbinsi Fernández-Triana \& Whitfield, sp. n. http://zoobank.org/4C754CF8-825E-42AE-99C0-D59F17DC178A http://species-id.net/wiki/Prasmodon_bobrobbinsi}

Figs 19-23, 100

Type locality. COSTA RICA, ACG, Alajuela Province, Sector Rincon Rain Forest, San Lucas, 320m, 10.91847, -85.30338.

Holotype. $q$ in CNC. Specimen labels: 1. DHJPAR0038915. 2. Voucher: D.H.Janzen \& W.Hallwachs, DB: http://janzen.sas.upenn.edu, Area de Conservación Guanacaste, COSTA RICA, 10-SRNP-40484.

Paratype. 1 q $1 \hat{\jmath}$ (CNC). COSTA RICA, ACG database codes: DHJPAR0040012, 09-SRNP-3496.

Description. Female. Body length $3.7-3.8 \mathrm{~mm}$ or $3.9-4.0 \mathrm{~mm}$. Fore wing length 3.9-4.0 $\mathrm{mm}$ or $4.1-4.2 \mathrm{~mm}$. Body color: mesosoma entirely yellow-orange (with the exception of a very small black spot on axillar complex), metasoma with brown to black areas covering most of mediotergites 4-7 (and also part of mediotergites 3 and 8) (Figs 19, 22, 23). Scape color: partially dark brown to black. Flagellomeres color: all flagellomeres brown to black (Figs 19, 21). Tegula and humeral complex color: tegula pale, humeral complex partially dark/partialy pale. Mesotibia color: entirely yellow. Metatibia color: posterior $0.1-0.3$ dark brown to black. Metatibia spurs color: yellow-orange. Metatarsus color: dark brown to black (except for anterior 0.7 or less of first metatarsomere). Fore wing color pattern: uniformly and entirely infumate (except for small hyaline area near veins $(\mathrm{RS}+\mathrm{M}) \mathrm{b}$ and $2 \mathrm{M})$. Fore wing veins color: all veins dark brown (Fig. 21). Pterostigma color: entirely dark brown. Hypostomal carina: highly raised (Fig. 20). Scutoscutellar sulcus: with 6 impressions (Fig. 22). Areolet height $\div$ vein $\mathrm{r}$ length (fore wing): $0.2 \times$. Hind wing subbasal cell: mostly with setae. Hind tarsal claws: with pectination uniform, teeth thick and relatively evenly spaced. Shape of mediotergite 1: distinctly narrowing posteriorly, width at posterior margin clearly less than width at anterior margin and median width (Fig. 23). Mediotergite 1 length $\div$ width at posterior margin 4.1-4.5 $\times$ or 4.6-5.0 $\times$. Mediotergite 2 width at posterior margin $\div$ length: $2.6-3.0 \times$. Ovipositor sheaths length: $0.5 \times$ as long as metatibia. 


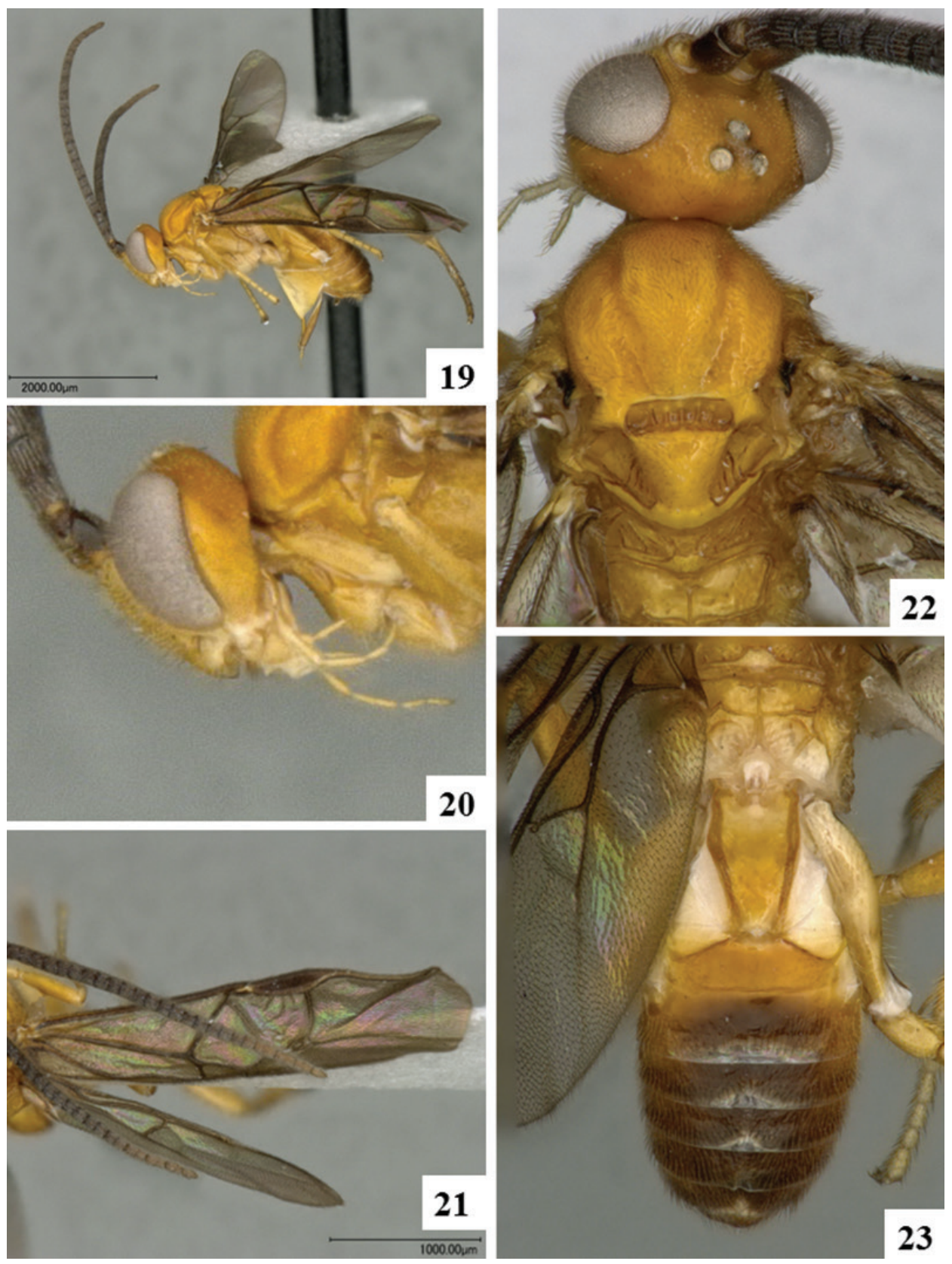

Figures 19-23. Prasmodon bobrobbinsi. 19 Habitus 20 Head and mesosoma (partially), lateral view $\mathbf{2}$ I Fore wing and antenna (partialy) $\mathbf{2 2}$ Head and mesosoma, dorsal view $\mathbf{2 3}$ Propodeum and metasoma, dorsal view.

Male. Morphologically similar to females.

Molecular data. Sequences in BOLD: 2, barcode compliant sequences: 2 (Fig. 105). Biology and ecology. Host: Crambidae, Palpita jairusalisDHJ01. 
Distribution. Costa Rica, ACG rain forest.

Etymology. This species is named in honour of Bob Robbins of the National Museum of Natural History, Smithsonian Institution, Washington, D.C, in recognition of his decades of taxonomic knowledge and support, contributing to understanding the species-level and higher taxonomy of the Lycaenidae of ACG, INBio and Costa Rica, as well as other parts of the world.

\section{Prasmodon dondavisi Fernández-Triana \& Whitfield, sp. n. http://zoobank.org/31AF25BD-0151-46EF-A980-1B9BF3BB339E http://species-id.net/wiki/Prasmodon_dondavisi}

Figs 24-28

Type locality. COSTA RICA, ACG, Alajuela Province, Sector Rincón Rain Forest, Jacobo, $461 \mathrm{~m}, 10.94076,-85.3177$.

Holotype. + in CNC. Specimen labels: 1. DHJPAR0040002. 2. Voucher: D.H.Janzen \& W.Hallwachs, DB: http://janzen.sas.upenn.edu, Area de Conservación Guanacaste, COSTA RICA, 09-SRNP-69586.

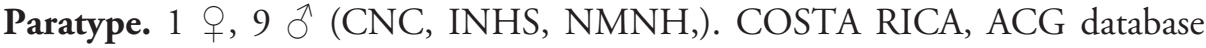
code: DHJPAR003990, DHJPAR0039992, DHJPAR0039994, DHJPAR0039995, DHJPAR0039998, DHJPAR004000, DHJPAR0040004, DHJPAR0040006, DHJPAR0040007, DHJPAR0040010.

Description. Female. Body length $5.3-5.4 \mathrm{~mm}$ or $5.5-5.6 \mathrm{~mm}$. Fore wing length $5.5-5.6 \mathrm{~mm}$ or $5.7-5.8 \mathrm{~mm}$. Body color: meso- and metasoma entirely yellow-orange (with the exception of a very small black spot on axillar complex) (Figs 24, 27, 28). Scape color: partially dark brown to black. Flagellomeres color: all flagellomeres brown to black (Fig. 24). Tegula and humeral complex color: both pale. Mesotibia color: entirely yellow. Metatibia color: posterior $0.1-0.3$ dark brown to black (Fig. 26). Metatibia spurs color: yellow-orange. Metatarsus color: dark brown to black (except for anterior 0.7 or less of first metatarsomere). Fore wing color pattern: uniformly and entirely infumate (except for small hyaline area near veins $(R S+M) b$ and $2 M)$. Fore wing veins color: all veins dark brown. Pterostigma color: entirely dark brown. Hypostomal carina: not raised (Fig. 25). Scutoscutellar sulcus: with 4 impressions (Fig. 27). Areolet height $\div$ vein $r$ length (fore wing): $0.3 \times$. Hind wing subbasal cell: mostly with setae. Hind tarsal claws: with pectination uniform, teeth thick and relatively evenly spaced. Shape of mediotergite 1: distinctly narrowing at around 0.5 its length, with both anterior and posterior margins clearly wider than its median width (Fig. 28). Mediotergite 1 length $\div$ width at posterior margin 3.1-3.5 $\times$ or 3.6-4.0 $\times$. Mediotergite 2 width at posterior margin $\div$ length: $3.1-3.5 \times$. Ovipositor sheaths length: $0.5 \times$ as long as metatibia.

Male. Morphologically similar to females.

Molecular data. Sequences in BOLD: 17, barcode compliant sequences: 15 (Fig. 105).

Biology and ecology. Hosts: Crambidae feeding on Apocynaceae: Prenesta Janzen196, Prenesta Janzen195, Prenesta scyllalisDHJ03. 

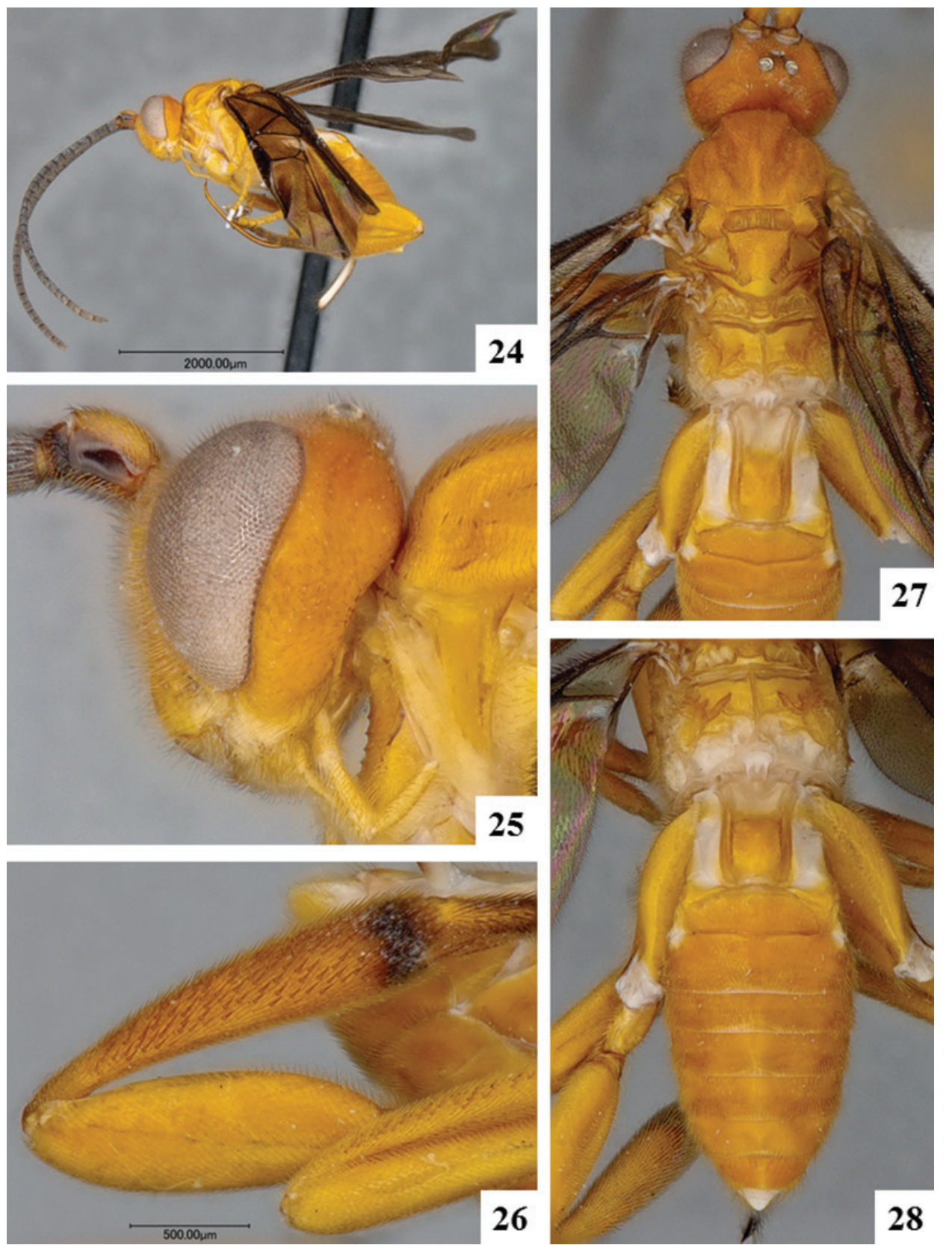

Figures 24-28. Prasmodon dondavisi. 24 Habitus 25 Head and mesosoma (partially), lateral view 26 Metatibia $\mathbf{2 7}$ Head and mesosoma, dorsal view 28 Propodeum and metasoma, dorsal view.

Distribution. Costa Rica, ACG rain forest.

Etymology. This species is named in honour of Don Davis of the National Museum of Natural History, Smithsonian Institution, Washington, D.C, in recognition 
of his decades of taxonomic knowledge and support, contributing to understanding the species-level and higher taxonomy of the Tineoidea and other small impossible moths of ACG, INBio and Costa Rica, as well as other parts of the world.

\section{Prasmodon eminens Nixon, 1965}

http://species-id.net/wiki/Prasmodon_eminens

Figs 95, 97

Prasmodon eminens Nixon, 1965: 206.

Type locality. PERU: Chanchamayo.

Holotype. ${ }^{\lambda}$, NHM (not examined).

Material examined. 8 , $18 \curvearrowright(\mathrm{BMNH}, \mathrm{CNC}$, INBio, INHS, NMNH), Costa Rica, ACG; $2 \AA$ (CNC), Ecuador, Pichincha, Rio Palenque, 160-200m.

Description. Female. Body length 4.9-5.0 mm, 5.1-5.2 mm, rarely 5.3-5.4 mm. Fore wing length 5.1-5.2 mm, 5.3-5.4 mm, rarely 5.5-5.6 mm. Body color: mesosoma entirely yellow-orange (with the exception of a very small black spot on axillar complex), metasoma with only small brown areas centrally on mediotergites 4-7 (usually only on mediotergires 5-6) (Fig. 95). Scape color: partially dark brown to black. Flagellomeres color: most flagellomeres brown to black, except for small apical area (F15-16, and occasionally apical half of F14) which is yellow-brown. Tegula and humeral complex color: both pale. Mesotibia color: posterior 0.1-0.2 dark brown to black. Metatibia color: posterior 0.1-0.3 dark brown to black (Fig. 95). Metatibia spurs color: dark brown to black. Metatarsus color: dark brown to black (except for anterior 0.7 or less of first metatarsomere) (Fig. 95). Fore wing color pattern: uniformly and entirely infumate (except for small hyaline area near veins $(\mathrm{RS}+\mathrm{M}) \mathrm{b}$ and $2 \mathrm{M})$. Fore wing veins color: all veins dark brown (Fig. 95). Pterostigma color: entirely dark brown. Hypostomal carina: not raised. Scutoscutellar sulcus: with 5 impressions or with 6 impressions. Areolet height $\div$ vein $r$ length (fore wing): $0.2 \times$. Hind wing subbasal cell: mostly without setae. Hind tarsal claws: with pectination uniform, teeth thick and relatively evenly spaced. Shape of mediotergite 1: distinctly narrowing posteriorly, width at posterior margin clearly less than width at anterior margin and median width (Fig. 95). Mediotergite 1 length $\div$ width at posterior margin 4.1-4.5 $\times$. Mediotergite 2 width at posterior margin $\div$ length: $3.1-3.5 \mathrm{x}$, rarely $2.6-3.0 \times$. Ovipositor sheaths length: $0.5 \times$ as long as metatibia or $0.6 \times$ as long as metatibia.

Male. As female but with flagellomeres fully dark brown to black.

Molecular data. Sequences in BOLD: 67, barcode compliant sequences: 61 (Fig. 105).

Biology and ecology. Hosts: Crambidae, Anarmodia nebulosalis, Asturodes fimbriauralisDHJ02, Bocchoris marucalis, Ceratocilia sixolalis, Desmia Janzen07, Desmia Solis19, Eulepte concordalis, Eulepte Janzen12, Eulepte Solis15, Mimophobetron pyropsalis, Omiodes fulvicauda, Omiodes humeralis, Pantographa suffusalis, Parastenia retractalis, Phostria mapetalis, Piletosoma thialis, Portentomorpha xanthialis, and Syllepte amandoDHJ02. 
Distribution. Widely distributed in rain forest in northern South America and Central America, including Brazil (Mardulyn and Whitfield 1999), Costa Rica, ACG rain forest (Valerio et al. 2005), Ecuador (this paper), and Peru (Nixon 1965).

Comments. This species was described by Nixon (1965) based on one male specimen. Valerio et al. (2005) extensively illustrated the species, including the cocoon and adults, but until now no detailed description of the female was available. DNA barcodes were made available recently by Smith et al. (2013). Host records are considerably expanded here, based on new data from the ongoing inventory of ACG parasitoids. Valerio et al. (2005) mentioned that this species shows some regional morphological variation and might well represent a complex of sibling species. Since we were not able to examine the holotype, or DNA barcode it, we infer from its morphological description that the ACG Prasmodon eminens is the same species.

\section{Prasmodon erenadupontae Braet \& Fernández-Triana, sp. $\mathbf{n}$. http://zoobank.org/D163BA78-BDE4-4F8E-B549-909751C9785A http://species-id.net/wiki/Prasmodon_erenadupontae}

Figs 29-34

Type locality. FRENCH GUIANA, Saul.

Holotype. + in MNHN. Specimen labels: 1. Guyane Francaise, Saul, 29.IX.2010, SEAG rec 2010.

Paratype. 2 $\uparrow, 3 \hat{O}$ (CNC, IRSNB). French Guiana, same locality than holotype, 17-26.ix.2010 (1 †, 1 §ै); Montagne de Kaw, Relais Patawa, ix.1999 (1 †); Nouragues Reserve, Inselberg, 16.x.2010 (1 §); Brazil, Mato Grosso, Sinop, xi.1975 (1 ठ).

Description. Female. Body length 5.3-5.4 mm, 5.5-5.6 mm or $5.7-5.8 \mathrm{~mm}$. Fore wing length $5.9-6.0 \mathrm{~mm}$ or $6.1-6.2 \mathrm{~mm}$. Body color: meso- and metasoma entirely yellow-orange (with the exception of a very small black spot on axillar complex) (Figs 29, 32, 34). Scape color: partially dark brown to black. Flagellomeres color: with relatively extense yellow area (at least including F13-15, and usually apical half of F11 and basal half of F16) (Fig. 30). Tegula and humeral complex color: both pale. Mesotibia color: entirely yellow. Metatibia color: posterior $0.1-0.3$ dark brown to black. Metatibia spurs color: yellow-orange. Metatarsus color: dark brown to black (except for anterior 0.7 or less of first metatarsomere) (Fig. 30). Fore wing color pattern: mostly infumate, but with pale area centrally which gives the wing a banded appearance (Figs 30, 33). Fore wing veins color: anterior $0.4-0.5$ of vein $3 \mathrm{M}$ (and usually part or all of veins $2 \mathrm{M}$ and $\mathrm{r}-\mathrm{m}$ ) yellow-orange, contrasting with all other veins which are dark brown (Fig. 33). Pterostigma color: entirely dark brown (Fig. 30). Hypostomal carina: highly raised (Fig. 31). Scutoscutellar sulcus: with 4 impressions (Fig. 32). Areolet height $\div$ vein $r$ length (fore wing): $0.2 \times$. Hind wing subbasal cell: mostly without setae. Hind tarsal claws: with pectination uniform, teeth thick and relatively evenly spaced. Shape of mediotergite 1: distinctly narrowing at around 0.5 its length, with both anterior and posterior margins clearly wider than its median 

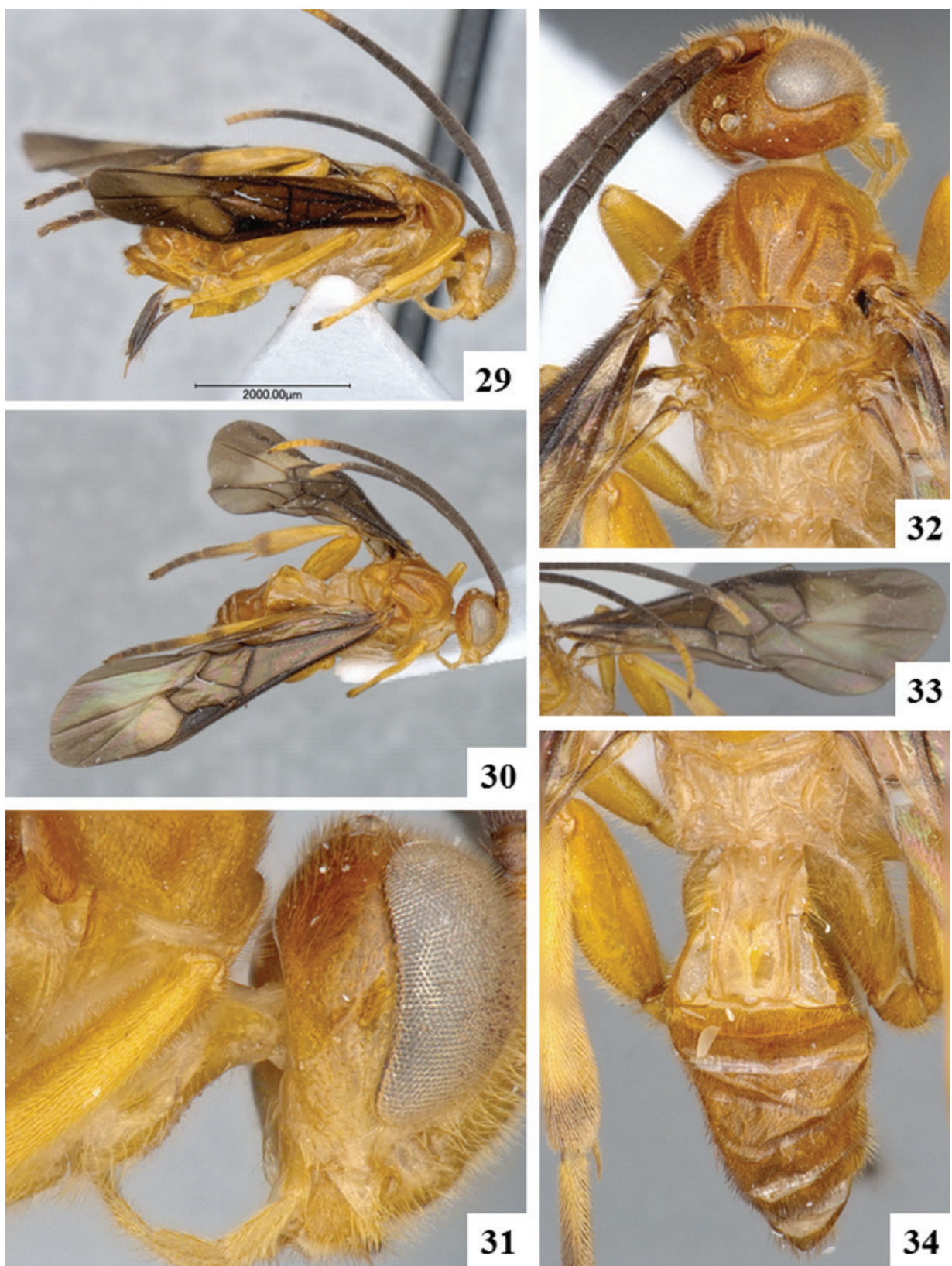

30

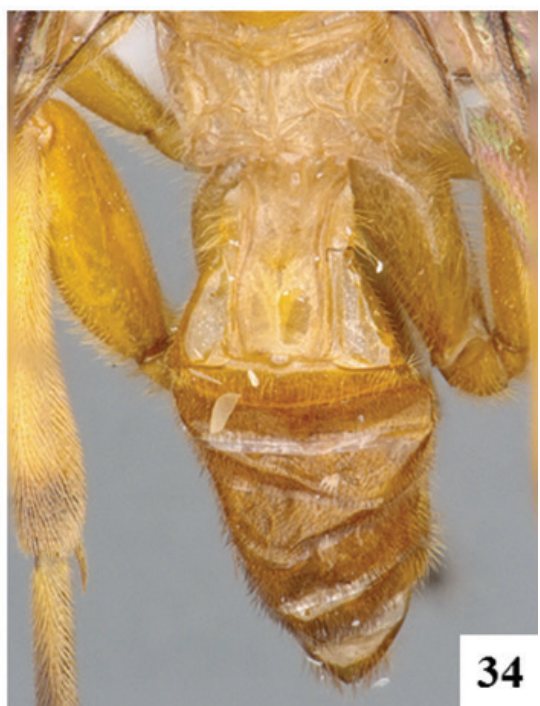

Figures 29-34. Prasmodon erenadupontae. 29 Habitus 30 Body, dorso-lateral view $\mathbf{3}$ I Head and mesosoma (partially), lateral view 32 Head and mesosoma, dorsal view 33 Fore wing $\mathbf{3 4}$ Propodeum and metasoma, dorsal view.

width (Fig. 34). Mediotergite 1 length $\div$ width at posterior margin 2.9-3.0 $\times$. Mediotergite 2 width at posterior margin $\div$ length: $3.6-4.0 \times$. Ovipositor sheaths length: $0.5 \times$ as long as metatibia. 
Male. Morphologically similar to females, but with antenna only yellow on flagellomeres 15-16 (females with flagellomeres 12-16 yellow).

Molecular data. Sequences in BOLD: 1, barcode compliant sequences: 1 .

Biology and ecology. Malaise trapped.

Distribution. Brazil, French Guiana.

Etymology. Named after Erena Dupont (the partner of YB) who shares his passion in entomology and for her strong support.

Comments. The only specimen with associated molecular data is the male paratype from Brazil.

\section{Prasmodon jobnbrowni Fernández-Triana \& Whitfield, sp. n. http://zoobank.org/F131E6A1-F5FF-414D-803F-7719C0E2A533 http://species-id.net/wiki/Prasmodon_johnbrowni}

Figs 35-39, 103

Type locality. COSTA RICA, ACG, Alajuela Province, Sector Rincon Rain Forest, Estación Llanura, 135m, 10.93332, -85.25331.

Holotype. $q$ in CNC. Specimen labels: 1. DHJPAR0035300. 2. Voucher: D.H.Janzen \& W.Hallwachs, DB: http://janzen.sas.upenn.edu, Area de Conservación Guanacaste, COSTA RICA, 09-SRNP-44366.

Paratype. 5 +, 2 (CNC, NMNH). COSTA RICA, ACG database code: DHJPAR0038172, DHJPAR0038174, DHJPAR0038175, DHJPAR0038177, DHJPAR0038922, DHJPAR0039993.

Description. Female. Body length 4.5-4.6 mm, 4.7-4.8 mm, 4.9-5.0 mm, rarely 5.1-5.2 mm. Fore wing length 4.9-5.0 mm, 5.1-5.2 mm, rarely 5.3-5.4 mm. Body color: meso- and metasoma entirely yellow-orange (with the exception of a very small black spot on axillar complex) (Figs 35, 38, 39). Scape color: partially dark brown to black. Flagellomeres color: all flagellomeres brown to black (Fig. 35). Tegula and humeral complex color: tegula pale, humeral complex dark. Mesotibia color: posterior 0.1-0.2 dark brown to black. Metatibia color: posterior 0.1-0.3 dark brown to black (Fig. 37). Metatibia spurs color: dark brown to black (Figs 35, 37). Metatarsus color: dark brown to black (except for anterior 0.7 or less of first metatarsomere). Fore wing color pattern: uniformly and entirely infumate (except for small hyaline area near veins $(\mathrm{RS}+\mathrm{M}) \mathrm{b}$ and $2 \mathrm{M})$. Fore wing veins color: all veins dark brown (Fig. 36). Pterostigma color: entirely dark brown (Fig. 38). Hypostomal carina: not raised. Scutoscutellar sulcus: with 6 impressions, rarely with 5 impressions. Areolet height $\div$ vein $r$ length (fore wing): $0.3 \times$. Hind wing subbasal cell: mostly without setae. Hind tarsal claws: with pectination uniform, teeth thick and relatively evenly spaced. Shape of mediotergite 1: distinctly narrowing posteriorly, width at posterior margin clearly less than width at anterior margin and median width (Fig. 39). Mediotergite 1 length $\div$ width at posterior margin 6.1-6.5 $\times$. Mediotergite 2 width at posterior margin $\div$ length: $2.6-3.0 \times$. Ovipositor sheaths length: $0.5 \times$ as long as metatibia or $0.6 \times$ as long as metatibia.

Male. Morphologically similar to females. 

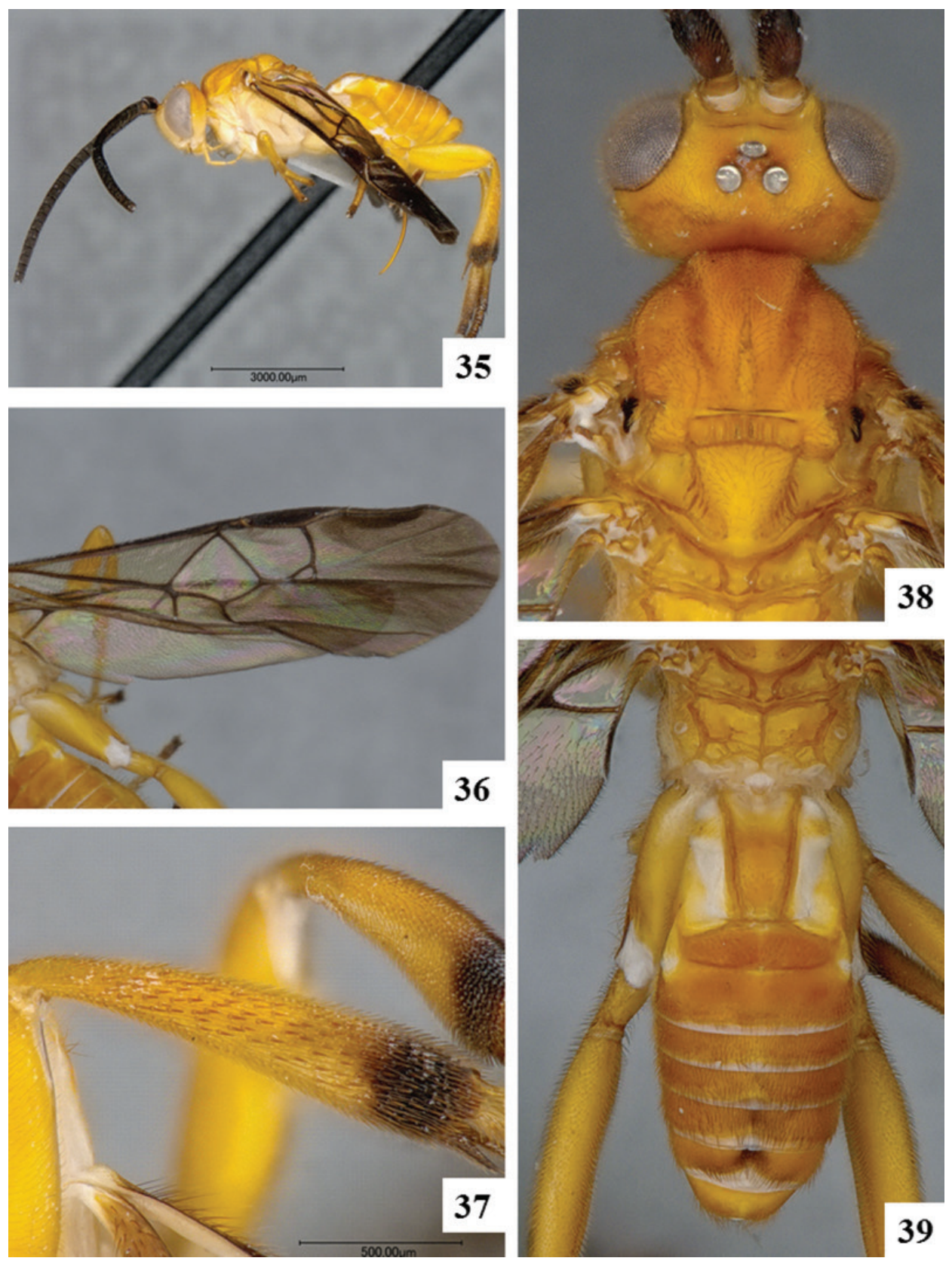

Figures 35-39. Prasmodon johnbrowni. 35 Habitus 36 Fore wing 37 Metatibia 38 Head and mesosoma, dorsal view 39 Propodeum and metasoma, dorsal view.

Molecular data. Sequences in BOLD: 12, barcode compliant sequences: 12 (Fig. 105). Biology and ecology. Hosts: Crambidae, Asturodes fimbriauralis DHJ02, Asturodes fimbriauralis, Eulepte alialis, Piletosoma thialis, Phostria Janzen05.

Distribution. Costa Rica, ACG rain forest. 
Etymology. This species is named in honour of John Brown of the SEL/USDA laboratory in the National Museum of Natural History, Smithsonian Institution, Washington, D.C, in recognition of his decades of taxonomic knowledge and support contributing to understanding the species-level and higher taxonomy of the Tortricoidea of ACG, INBio and Costa Rica, as well as other parts of the world.

\section{Prasmodon masoni Fernández-Triana $\&$ Whitfield, sp. $\mathbf{n}$.}

http://zoobank.org/39CD8542-C231-4CDB-A67B-57782406696B http://species-id.net/wiki/Prasmodon_masoni

Figs $40-45$

Type locality. BRAZIL, Mato Grosso, Sinop.

Holotype. + in CNC. Specimen labels: 1. BRAZIL, Mato Grosso, Sinop, X.1974., M. Alvarenga, Mal. Trap. 2. DNA Voucher, CNCHYM 01962.

Paratype. 1 9, 6 \# M (CNC), Brazil, same locality than holtype, collecting dates: x.1974, x.1975, ii.1976.

Other material examined. 1 (CNC) from Brazil, Amazonas, Estirar de Ecuador, Rio Javari, ix.1979.

Description. Female. Body length 3.5-3.6 mm, rarely 3.9-4.0 mm. Fore wing length $3.7-3.8 \mathrm{~mm}$, rarely $4.1-4.2 \mathrm{~mm}$. Body color: meso- and metasoma entirely yellow-orange (with the exception of a very small black spot on axillar complex) (Figs 40, 44, 45). Scape color: partially dark brown to black (Fig. 44). Flagellomeres color: all flagellomeres brown to black (Figs 40-42). Tegula and humeral complex color: both pale. Mesotibia color: entirely yellow. Metatibia color: entirely yellow (Fig. 43). Metatibia spurs color: yellow-orange. Metatarsus color: entirely yellow-white (Fig. 43). Fore wing color pattern: hyaline. Fore wing veins color: all veins dark brown. Pterostigma color: entirely dark brown. Hypostomal carina: not raised (Fig. 42). Scutoscutellar sulcus: with 4 impressions (Fig. 44). Areolet height $\div$ vein $r$ length (fore wing): $0.2 \mathrm{x}$, rarely $0.3 \times$. Hind wing subbasal cell: mostly with setae (Figs 44, 45). Hind tarsal claws: with pectination (teeth) very irregular in spacing and length. Shape of mediotergite 1: distinctly narrowing posteriorly, width at posterior margin clearly less than width at anterior margin and median width (Fig. 45). Mediotergite 1 length $\div$ width at posterior margin $4.1-4.5 \times$ or $4.6-5.0 \times$. Mediotergite 2 width at posterior margin $\div$ length: 2.6-3.0 $\times$. Ovipositor sheath length: $0.3 \times$ as long as metatibia.

Male. Morphologically similar to female.

Molecular data. No DNA barcodes were obtained for this species.

Biology and ecology. Malaise-trapped.

Distribution. Brazil, Mato Grosso.

Etymology. Named after William R. M. Mason, the greatest Canadian expert on Microgastrinae. The holotype and paratype specimen had a label written by Mason, who considered them to represent a new species (Mason, 1981), which remained undescribed until now. 

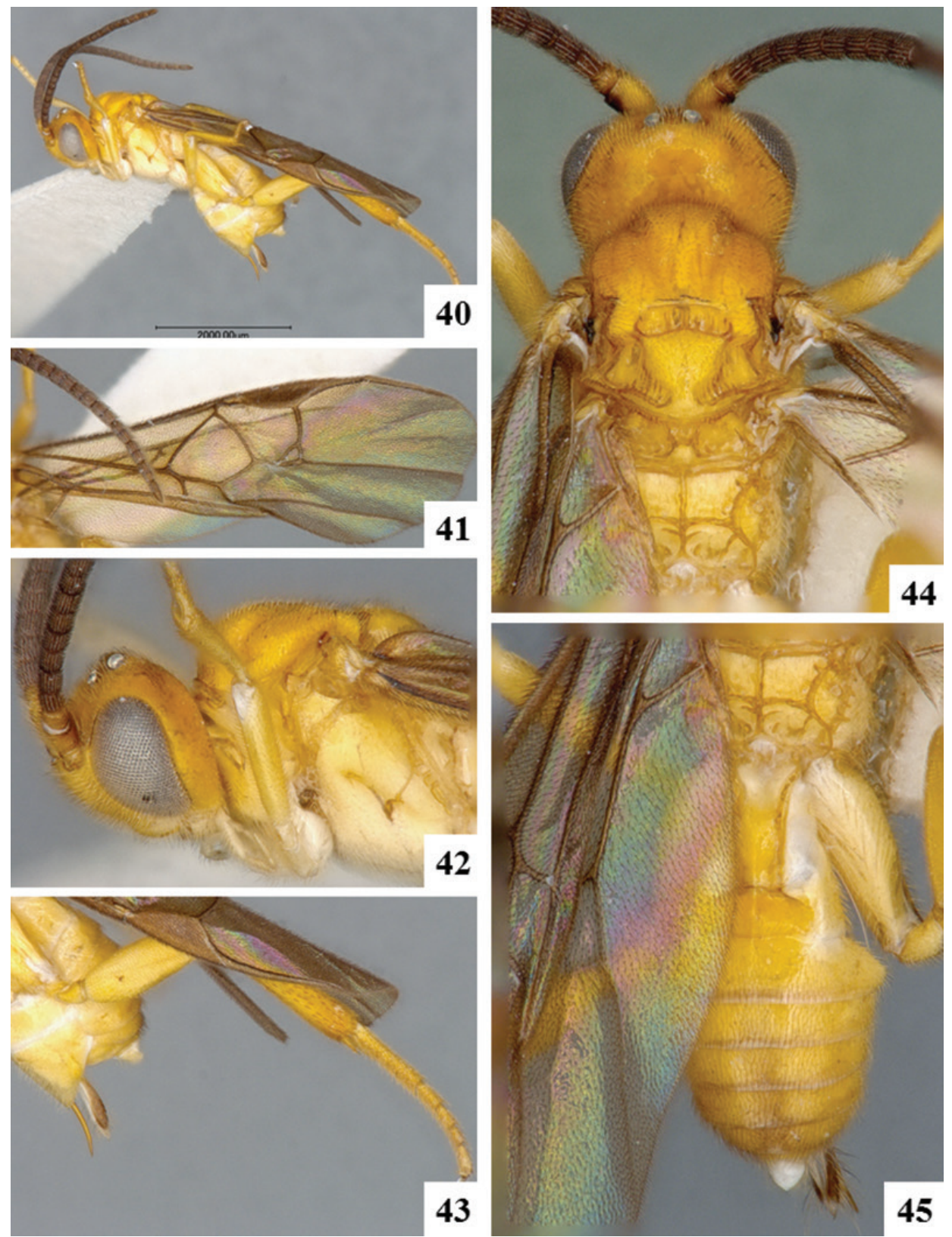

Figures 40-45. Prasmodon masoni. $\mathbf{4 0}$ Habitus $\mathbf{4}$ I Fore wing $\mathbf{4 2} \mathrm{Head}$ and mesosoma (partially), lateral view 43 Hypopygium, ovipositor, ovipositor sheaths, and hind leg (partially), lateral view $44 \mathrm{Head}$ and mesosoma, dorsal view 45 Propodeum and metasoma, dorsal view.

Comments. We have included here one female from a locality (Rio Javari, Brazil) far from the type locality (Sinop, where all other specimens of the species were collected). The female from Rio Javari resembles the female holotype and another female 
paratype, but was not included as part of the paratype series in case it is eventually found to represent a different species. We also studied another $15 \mathrm{CNC}$ male specimens of Prasmodon that were collected in Mato Grosso. They seem to represent 2-3 additional species. However, no molecular or host information is available for those specimens, and the morphological data are inadequate to properly describe them at present.

\section{Prasmodon mikepoguei Fernández-Triana \& Whitfield, sp. n.}

http://zoobank.org/A2BBA863-E45D-4A6C-AAFD-217CE7FABBC2 http://species-id.net/wiki/Prasmodon_mikepoguei

Figs 46-51, 96

Type locality. COSTA RICA, ACG, Guanacaste Province, Sector Pitilla, Leonel, 510m, 10.99637, -85.40195 .

Holotype. $\delta$ in CNC. Specimen labels: 1. DHJPAR0038222. 2. Voucher: D.H.Janzen \& W.Hallwachs, DB: http://janzen.sas.upenn.edu, Area de Conservación Guanacaste, COSTA RICA, 09-SRNP-72161.

Description. Male. Body length 3.7-3.8 mm. Fore wing length 4.1-4.2 mm. Body color: meso- and metasoma with brown to black areas, metasoma with most of tergites 2-8 dark brown (Figs 46, 50, 51). Scape color: partially dark brown to black. Flagellomeres color: all flagellomeres brown to black (Fig. 47). Tegula and humeral complex color: tegula pale, humeral complex dark. Mesotibia color: entirely yellow. Metatibia color: posterior $0.1-0.3$ dark brown to black (Fig. 49). Metatibia spurs color: yelloworange. Metatarsus color: dark brown to black (except for anterior 0.7 or less of first metatarsomere). Fore wing color pattern: uniformly and entirely infumate (except for small hyaline area near veins $(\mathrm{RS}+\mathrm{M}) \mathrm{b}$ and $2 \mathrm{M})$. Fore wing veins color: all veins dark brown. Pterostigma color: entirely dark brown. Hypostomal carina: not raised. Scutoscutellar sulcus: with 4 impressions (Fig. 50). Hind wing subbasal cell: mostly with setae (Figs 48, 50, 51). Hind tarsal claws: with pectination uniform, teeth thick and relatively evenly spaced. Shape of mediotergite 1: distinctly narrowing posteriorly, width at posterior margin clearly less than width at anterior margin and median width (Fig. 51).

Female. Unknown.

Molecular data. Sequences in BOLD: 4, barcode compliant sequences: 4 (Fig. 105).

Biology and ecology. Hosts: Elachistidae, Chlamydastis tryphon, Antaeotricha BioLep42, Antaeotricha Janzen07.

Distribution. Costa Rica, ACG rain forest.

Etymology. This species is named in honour of Mike Pogue of the SEL/USDA laboratory in the National Museum of Natural History, Smithsonian Institution, Washington, D.C, in recognition of his decades of taxonomic knowledge and support contributing to understanding the species-level and higher taxonomy of the Notodontidae and Noctuidae of ACG, INBio and Costa Rica, as well as other parts of the world. 

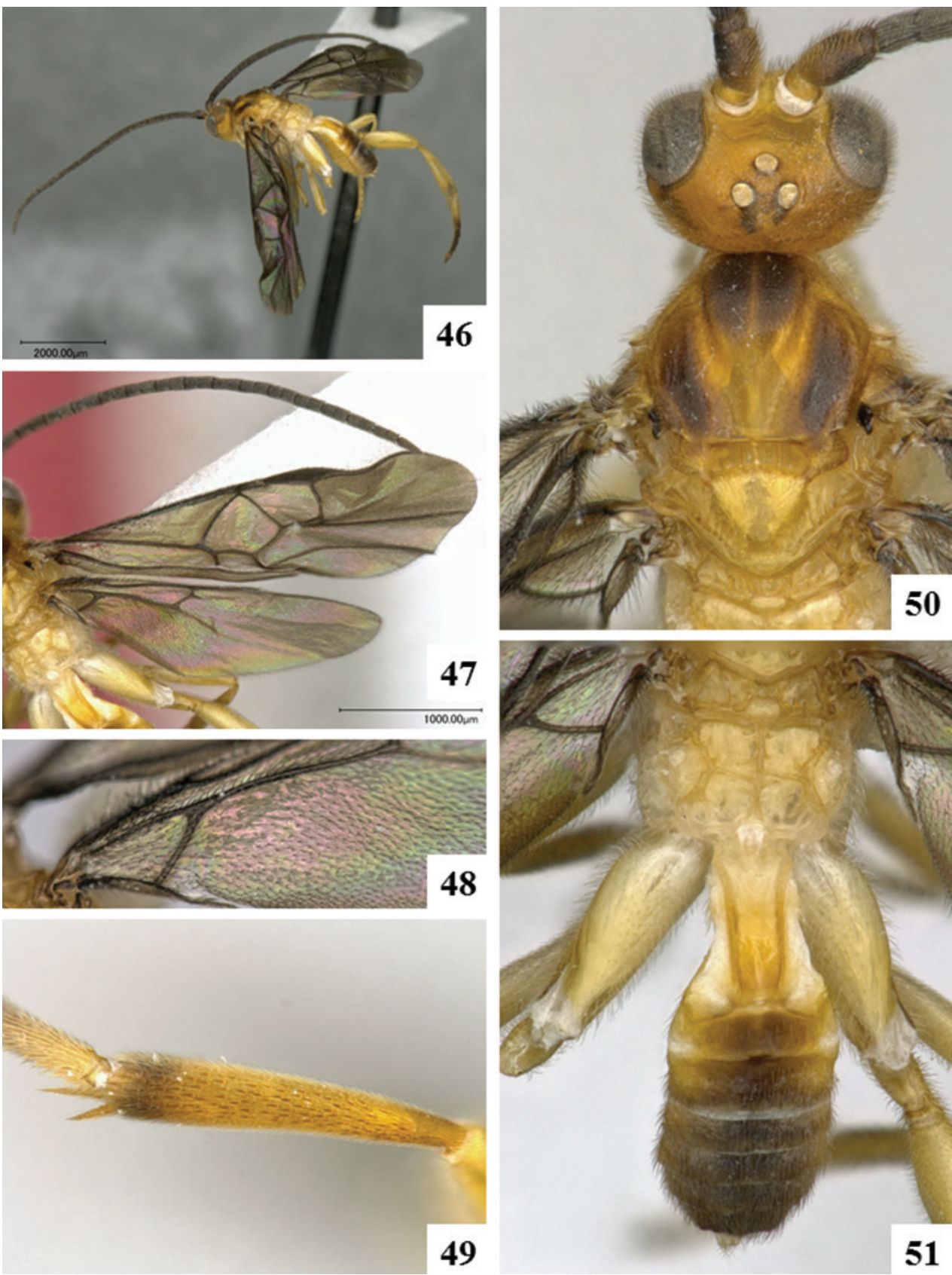

Figures 46-5 I. Prasmodon mikepoguei. 46 Habitus 47 Fore wing 48 Detail of the hind wing 49 Metatibia 50 Head and mesosoma, dorsal view $\mathbf{5}$ I Propodeum and metasoma, dorsal view. 


\section{Prasmodon nixoni Fernández-Triana \& Whitfield, sp. n. \\ http://zoobank.org/989FD927-8A4A-4582-A69D-DE20AFA9841D \\ http://species-id.net/wiki/Prasmodon_nixoni}

Figs 52-58

Type locality. PERU, Loreto, Iquitos, Barillal.

Holotype. + in CNC. Specimen labels: 1. PERU: Loreto, Iquitos, Barillal, 10.ii.1984, L. Huggert. 2. DNA Voucher, CNCHYM 01957.

Paratype. 1 ( (IRSNB). French Guiana, Montagne de Kaw, Relais Patawa, v.2001.

Description. Female. Body length $4.3-4.4 \mathrm{~mm}$ or $4.5-4.6 \mathrm{~mm}$. Fore wing length $4.5-4.6 \mathrm{~mm}$ or $4.7-4.8 \mathrm{~mm}$. Body color: meso- and metasoma entirely yellow-orange (with the exception of a very small black spot on axillar complex) (Figs 52, 56, 58). Scape color: partially dark brown to black. Flagellomeres color: with relatively extense yellow area (at least including F13-15, and usually apical half of F11 and basal half of F16) (Figs 52-54). Tegula and humeral complex color: both pale. Mesotibia color: entirely yellow. Metatibia color: entirely yellow (Fig. 55). Metatibia spurs color: yellow-orange. Metatarsus color: dark brown to black (except for anterior 0.7 or less of first metatarsomere). Fore wing color pattern: hyaline. Fore wing veins color: all veins dark brown (Fig. 53). Pterostigma color: entirely dark brown. Hypostomal carina: not raised (Fig. 57). Scutoscutellar sulcus: with 6 impressions (Fig. 56). Areolet height $\div$ vein $r$ length (fore wing): $0.2 \times$. Hind wing subbasal cell: mostly with setae. Hind tarsal claws: with 1 or 2 teeth on anterior 0.3 of claw. Shape of mediotergite 1: distinctly narrowing posteriorly, width at posterior margin clearly less than width at anterior margin and median width (Fig. 58). Mediotergite 1 length $\div$ width at posterior margin 4.6-5.0 $\times$. Mediotergite 2 width at posterior margin $\div$ length: 3.6-4.0 $\times$. Ovipositor sheaths length: $0.5 \times$ as long as metatibia.

Male. Unknown.

Molecular data. Sequences in BOLD: 1, barcode compliant sequences: 0 .

Biology and ecology. Malaise-trapped.

Distribution. French Guiana, Peru.

Etymology. Named to honour Gilbert E. J. Nixon, one of the great British experts on Microgastrinae and the first person to describe a species of Prasmodon.

\section{Prasmodon paulgoldsteini Fernández-Triana \& Whitfield, sp. n.} http://zoobank.org/3AD73865-F1F1-415F-9DDB-41BFAA5D0E1A http://species-id.net/wiki/Prasmodon_paulgoldsteini

Figs 59-63

Type locality. COSTA RICA, Guanacaste, ACG, Sector Santa Rosa, Bosque San Emilio, 300m, 10.84389, -85.61384.

Holotype. $\delta$ in CNC. Specimen labels: 1. DHJPAR0013126.

Paratypes. $4 \pi$ (CNC, NMNH). COSTA RICA, ACG database codes: DHJPAR0013131, DHJPAR0013124, DHJPAR0013125, DHJPAR0013132. 

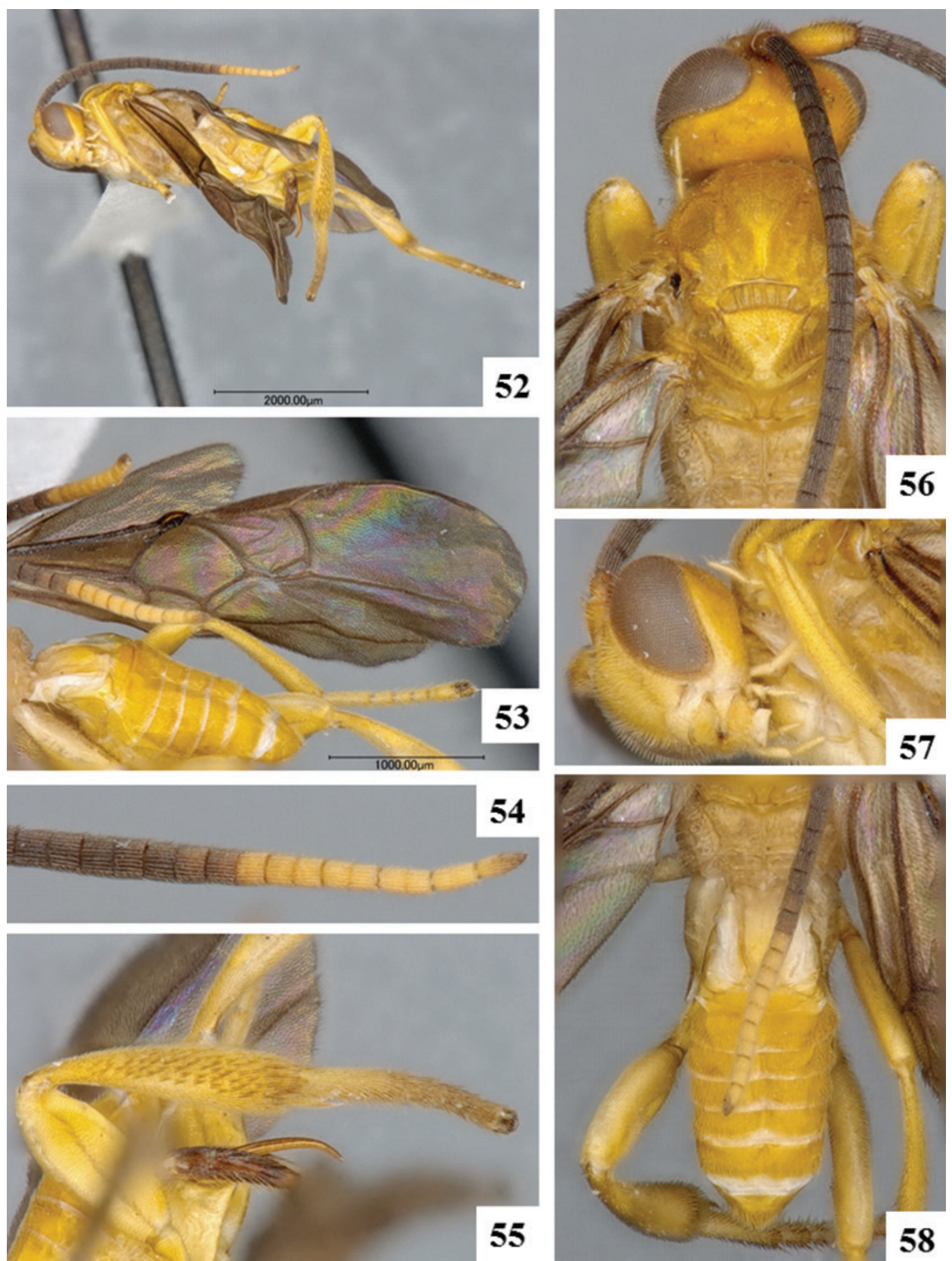

54

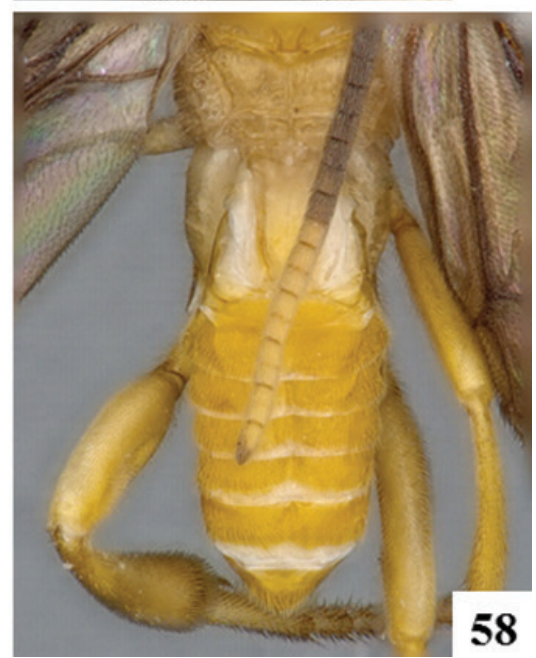

Figures 52-58. Prasmodon nixoni. 52 Habitus 53 Fore wing 54 Antenna (partially) 55 Metatibia 56 Head and mesosoma, dorsal view $\mathbf{5 7}$ Head and mesosoma (partially), lateral view $\mathbf{5 8}$ Propodeum and metasoma, dorsal view.

Description. Male. Body length 4.1-4.2 mm. Fore wing length $4.3-4.4 \mathrm{~mm}$. Body color: mesosoma entirely yellow-orange (with the exception of a very small black spot on axillar complex), metasoma with only small brown areas centrally on medioter- 

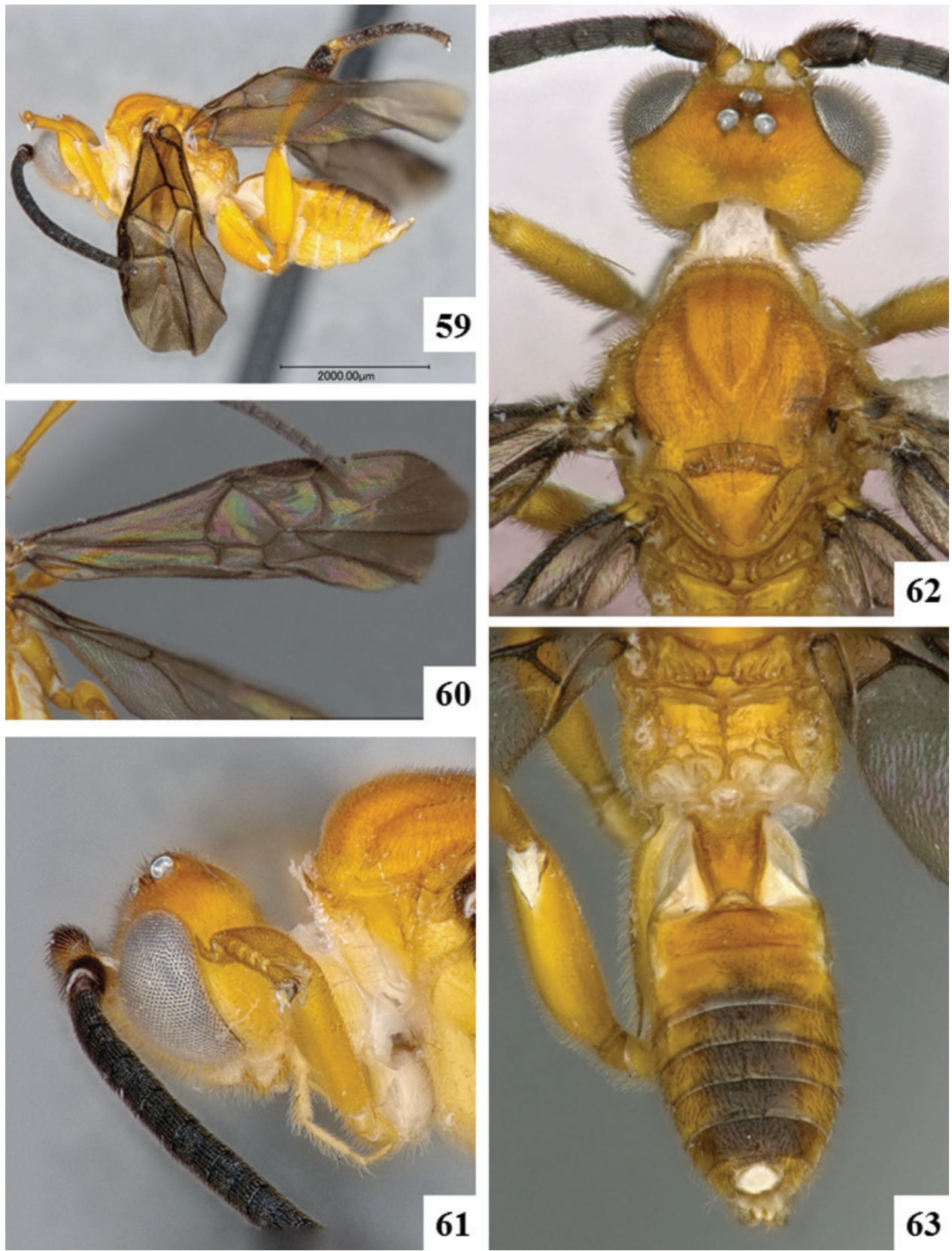

Figures 59-63. Prasmodon paulgoldsteini. 59 Habitus 60 Fore wing 61 Head and mesosoma (partially), lateral view 62 Head and mesosoma, dorsal view 63 Propodeum and metasoma, dorsal view.

gites 4-7 (usually only on mediotergires 5-6) (Figs 62, 63). Scape color: partially dark brown to black. Flagellomeres color: all flagellomeres brown to black (Fig. 59). Tegula and humeral complex color: tegula pale, humeral complex partially dark/partially pale. 
Mesotibia color: entirely yellow. Metatibia color: posterior $0.1-0.3$ dark brown to black (Fig. 59). Metatibia spurs color: yellow-orange. Metatarsus color: dark brown to black (except for anterior 0.7 or less of first metatarsomere). Fore wing color pattern: uniformly and entirely infumate (except for small hyaline area near veins $(R S+M) b$ and $2 \mathrm{M}$ ). Fore wing veins color: all veins dark brown (Fig. 60). Pterostigma color: entirely dark brown. Hypostomal carina: highly raised. Scutoscutellar sulcus: with 5 impressions or with 6 impressions. Hind wing subbasal cell: mostly with setae. Hind tarsal claws: with pectination (teeth) very irregular in spacing and length. Shape of mediotergite 1: distinctly narrowing posteriorly, width at posterior margin clearly less than width at anterior margin and median width (Fig. 63).

Female. Unknown.

Molecular data. Sequences in BOLD: 6, barcode compliant sequences: 6 (Fig. 105).

Biology and ecology. Malaise trapped.

Distribution. Costa Rica, ACG.

Comments. At present this species is only known from males, but it was described because of clear morphological and molecular differences with the other species within the genus.

Etymology. This species is named in honour of Paul Goldstein working in the SEL/USDA laboratory in the National Museum of Natural History, Smithsonian Institution, Washington, D.C, and with the Department of Entomology of the University of Maryland, in recognition of his intense effort to solve the Desmia (Crambidae, Spilomelinae) many-species taxonomic puzzle for ACG.

\section{Prasmodon scottmilleri Fernández-Triana $\&$ Whitfield, sp. n.}

http://zoobank.org/8BCFA3EE-53D0-4582-AE6B-A31D544DE9B1

http://species-id.net/wiki/Prasmodon_scottmilleri

Figs 64-69, 98

Type locality. COSTA RICA, ACG, Alajuela Province, Sector Rincon Rain Forest, Camino Albergue Oscar, 560m, 10.87741, -85.32363.

Holotype. + in CNC. Specimen labels: 1. DHJPAR0043031. 2. Voucher: D.H.Janzen \& W.Hallwachs, DB: http://janzen.sas.upenn.edu, Area de Conservación Guanacaste, COSTA RICA, 11-SRNP-610.

Paratype. $1 \widehat{\partial}$ (CNC). COSTA RICA, ACG database code: DHJPAR0038914.

Description. Female. Body length 3.9-4.0 mm. Fore wing length $4.5-4.6 \mathrm{~mm}$. Body color: mesosoma entirely yellow-orange (with the exception of a very small black spot on axillar complex), metasoma with brown to black areas covering most of mediotergites 4-7 (and also part of mediotergites 3 and 8). Scape color: partially dark brown to black. Flagellomeres color: most flagellomeres brown to black, except for small subapical area (F14-15) which is yellow-brown (Figs 64, 67). Tegula and humeral complex color: tegula pale, humeral complex partially dark/partialy pale. Mesotibia color: posterior $0.1-0.2$ dark brown to black. Metatibia color: posterior $0.1-0.3$ 

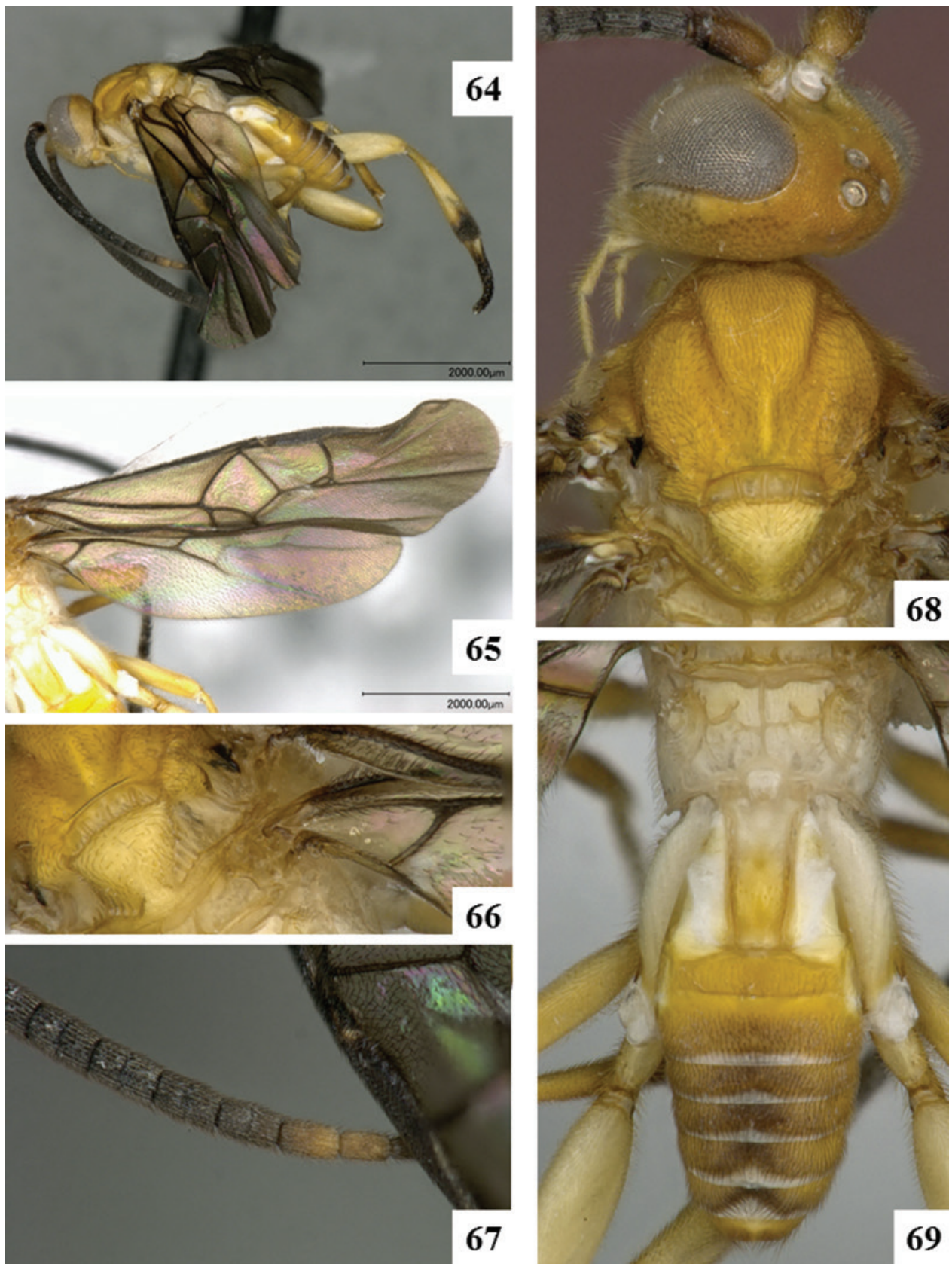

Figures 64-69. Prasmodon scottmilleri. 64 Habitus 65 Fore wing 66 Detail of the hind wing 67 Antenna (partially) $68 \mathrm{Head}$ and mesosoma, dorsal view 69 Propodeum and metasoma, dorsal view.

dark brown to black (Fig. 64). Metatibia spurs color: yellow-orange. Metatarsus color: dark brown to black (except for anterior 0.7 or less of first metatarsomere) (Fig. 64). Fore wing color pattern: uniformly and entirely infumate (except for small hyaline area 
near veins $(\mathrm{RS}+\mathrm{M}) \mathrm{b}$ and $2 \mathrm{M})$. Fore wing veins color: all veins dark brown (Fig. 65). Pterostigma color: entirely dark brown. Hypostomal carina: highly raised. Scutoscutellar sulcus: with 4 impressions. Areolet height $\div$ vein $r$ length (fore wing): $0.15 \times$ or less. Hind wing subbasal cell: mostly without setae (Figs 65, 69). Hind tarsal claws: with pectination (teeth) very irregular in spacing and length. Shape of mediotergite 1: distinctly narrowing posteriorly, width at posterior margin clearly less than width at anterior margin and median width (Fig. 69). Mediotergite 1 length $\div$ width at posterior margin 3.6-4.0 $\times$. Mediotergite 2 width at posterior margin $\div$ length: $3.6-4.0 \times$. Ovipositor sheaths length: $0.6 \times$ as long as metatibia.

Male. As female but with flagellomeres fully dark brown to black.

Molecular data. Sequences in BOLD: 6, barcode compliant sequences: 5 (Fig. 105).

Biology and ecology. Hosts: Crambidae, four species of Omiodes and Triuncidia eupalusalisDHJ02.

Distribution. Costa Rica, ACG rain forest.

Etymology. This species is named in honour of Scott Miller of the National Museum of Natural History, Smithsonian Institution, Washington, D.C, in recognition of his decades of taxonomic knowledge and support contributing to understanding the species-level and higher taxonomy of the Dalceridae, Lasiocampidae, and other fat fuzzy moths of ACG, INBio and Costa Rica, as well as other parts of the world, and administrating Lepidoptera biodiversity development at the Smithsonian Institution.

\section{Prasmodon silvatlanticus Fernández-Triana \& Whitfield, sp. n. http://zoobank.org/A9F2150A-1D0C-402E-9945-FC21A1663857 http://species-id.net/wiki/Prasmodon_silvatlanticus}

Figs 70-75

Type locality. BRAZIL, Est. Rio de Janeiro, Silva Jardim.

Holotype. + in CNC. Specimen labels: 1. BRAZIL, Est. Rio de Janeiro, Silva Jardim, viii.1974, F. M. Oliveira. 2. DNA Voucher CNCHYM 01960.

Description. Female. Body length 4.1-4.2 mm. Fore wing length 4.5-4.6 mm. Body color: mesosoma entirely yellow-orange (with the exception of a very small black spot on axillar complex), metasoma with brown to black areas covering most of mediotergites 4-7 (and also part of mediotergites 3 and 8). Scape color: partially dark brown to black. Flagellomeres color: most flagellomeres brown to black, except for small subapical area (F14 and apical half of F13) which is yellow-brown (Figs 70, 74). Tegula and humeral complex color: tegula pale, humeral complex dark. Mesotibia color: entirely yellow. Metatibia color: posterior $0.1-0.3$ dark brown to black (Figs 70, 72). Metatibia spurs color: yellow-orange. Metatarsus color: dark brown to black (except for anterior 0.7 or less of first metatarsomere) (Fig. 70). Fore wing color pattern: hyaline. Fore wing veins color: all veins dark brown (Fig. 71). Pterostigma color: entirely dark brown. Hypostomal carina: highly raised (Fig. 72). Scutoscutellar sulcus: with 6 impressions. Areolet height $\div$ vein $\mathrm{r}$ length (fore wing): $0.15 \times$ or less. Hind 

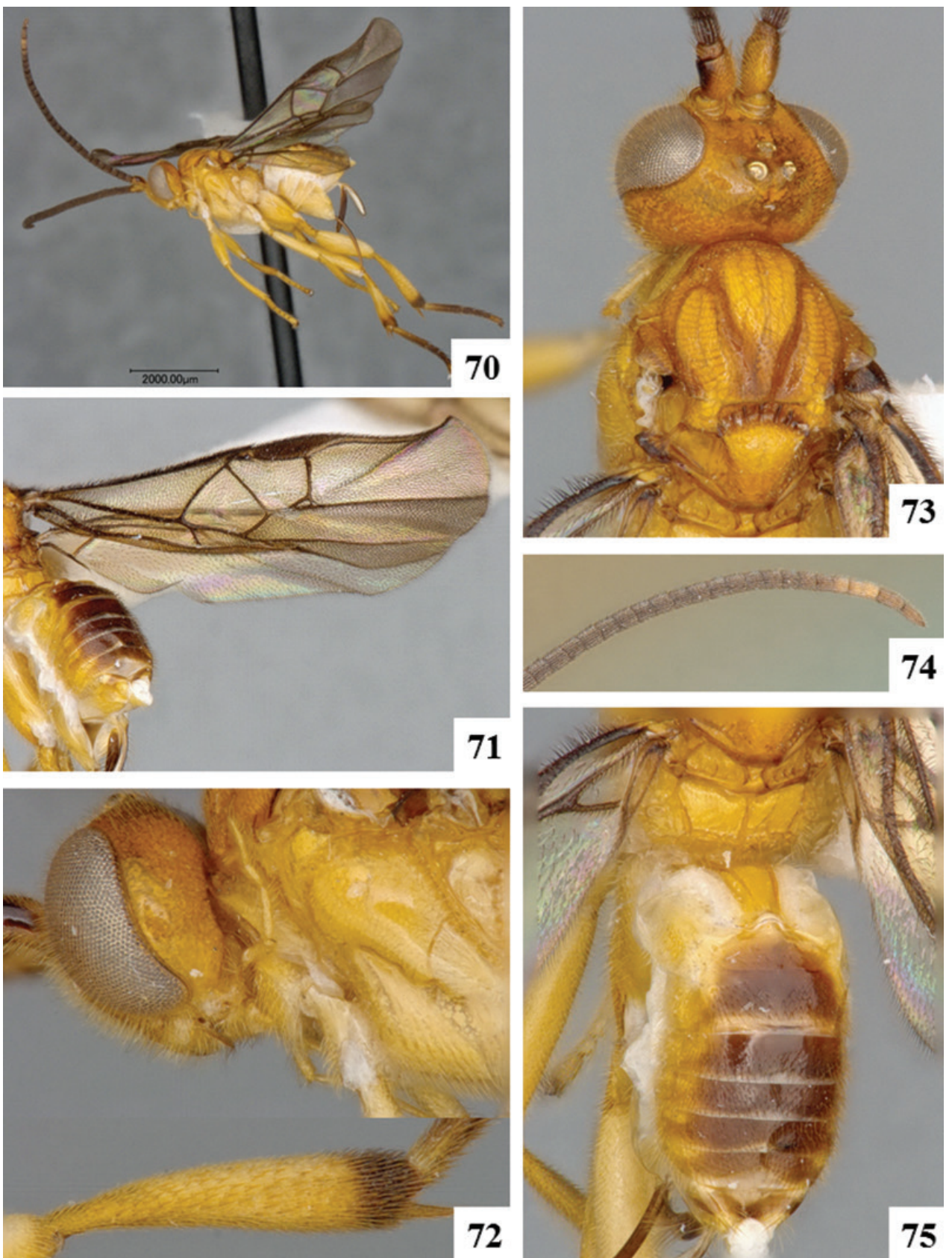

74

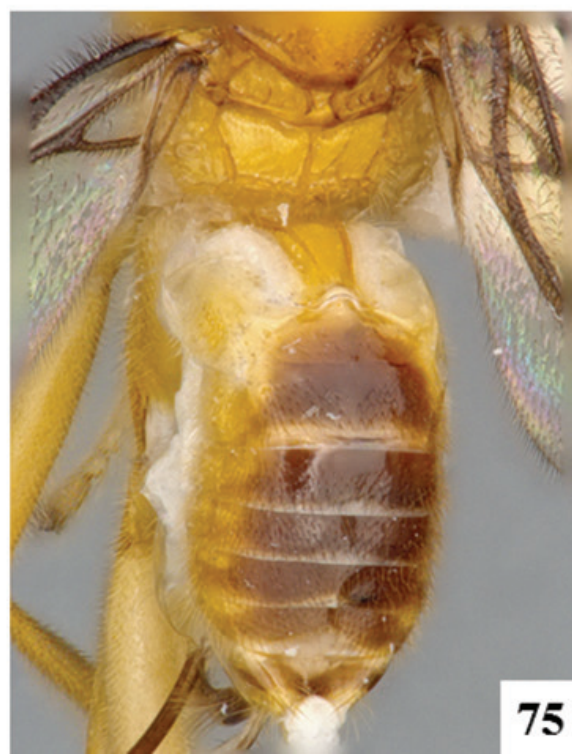

Figures 70-75. Prasmodon silvatlanticus. 70 Habitus 71 Fore wing $72 \mathrm{Head}$ and mesosoma (partially), lateral view $\mathbf{7 3} \mathrm{Head}$ and mesosoma, dorsal view $\mathbf{7 4}$ Antenna $\mathbf{7 5}$ Propodeum and metasoma, dorsal view.

wing subbasal cell: mostly without setae. Hind tarsal claws: with pectination uniform, teeth thick and relatively evenly spaced. Shape of mediotergite 1: distinctly narrowing posteriorly, width at posterior margin clearly less than width at anterior margin and 
median width (Fig. 75). Mediotergite 1 length $\div$ width at posterior margin 5.6-6.0 $\times$. Mediotergite 2 width at posterior margin $\div$ length: 2.6-3.0 $\times$. Ovipositor sheaths length: $0.6 \times$ as long as metatibia.

Male. Unknown.

Molecular data. Sequences in BOLD: 1, barcode compliant sequences: 0 .

Biology and ecology. Unknown.

Distribution. Brazil, Rio de Janeiro.

Etymology. From Latin "silva"="forest" and "atlanticus"=Atlantic, referring to the Atlantic Forest biome, where this and other species of Prasmodon have been found. The first part of the name also refers to the type locality (Silva Jardim).

Comments. The only known specimen was considered by Mason (1981) to be a new species, which remained undescribed until now.

\section{Prasmodon subfuscus Fernández-Triana $\&$ Whitfield, sp. $\mathbf{n}$. http://zoobank.org/8BD6E416-FF8F-4FDC-8581-9C97B061BA2A http://species-id.net/wiki/Prasmodon_subfuscus}

Figs $76-81$

Type locality. BRAZIL, Rio de Janeiro, Silva Jardim.

Holotype. \#1 in CNC. Specimen labels: 1. BRAZIL, Est. Rio de Janeiro, Silva Jardim, viii.1974, F. M. Oliveira. 2. DNA Voucher CNCHYM 01961.

Description. Male. Body length 4.5-4.6 mm. Fore wing length $5.1-5.2 \mathrm{~mm}$. Body color: meso- and metasoma with brown to black areas, metasoma with most of tergites 2-8 dark brown (Figs 77, 80, 81). Scape color: partially dark brown to black (Fig. 79). Flagellomeres color: all flagellomeres brown to black (Fig. 76). Tegula and humeral complex color: tegula pale, humeral complex dark. Metatibia color: posterior 0.1-0.3 dark brown to black (Fig. 78). Metatibia spurs color: yellow-orange (Fig. 78). Fore wing color pattern: uniformly and entirely infumate (except for small hyaline area near veins $(\mathrm{RS}+\mathrm{M}) \mathrm{b}$ and $2 \mathrm{M})$. Fore wing veins color: all veins dark brown (Fig. 77). Pterostigma color: entirely dark brown. Hypostomal carina: highly raised (Fig. 79). Scutoscutellar sulcus: with 5 impressions. Hind wing subbasal cell: mostly without setae. Hind tarsal claws: with pectination (teeth) very irregular in spacing and length. Shape of mediotergite 1: distinctly narrowing posteriorly, width at posterior margin clearly less than width at anterior margin and median width (Fig. 81).

Female. Unknown.

Molecular data. Sequences in BOLD: 1, barcode compliant sequences: 0 .

Biology and ecology. Unknown.

Distribution. Brazil, Rio de Janeiro.

Etymology. From Latin "subfuscus"=brownish, referring to the extensive brown coloration of the species, by far the least yellow of all known species of Prasmodon.

Comments. This species, only known from a single male, was collected in the same locality and on the same date as Prasmodon silvatlanticus, which is known only 

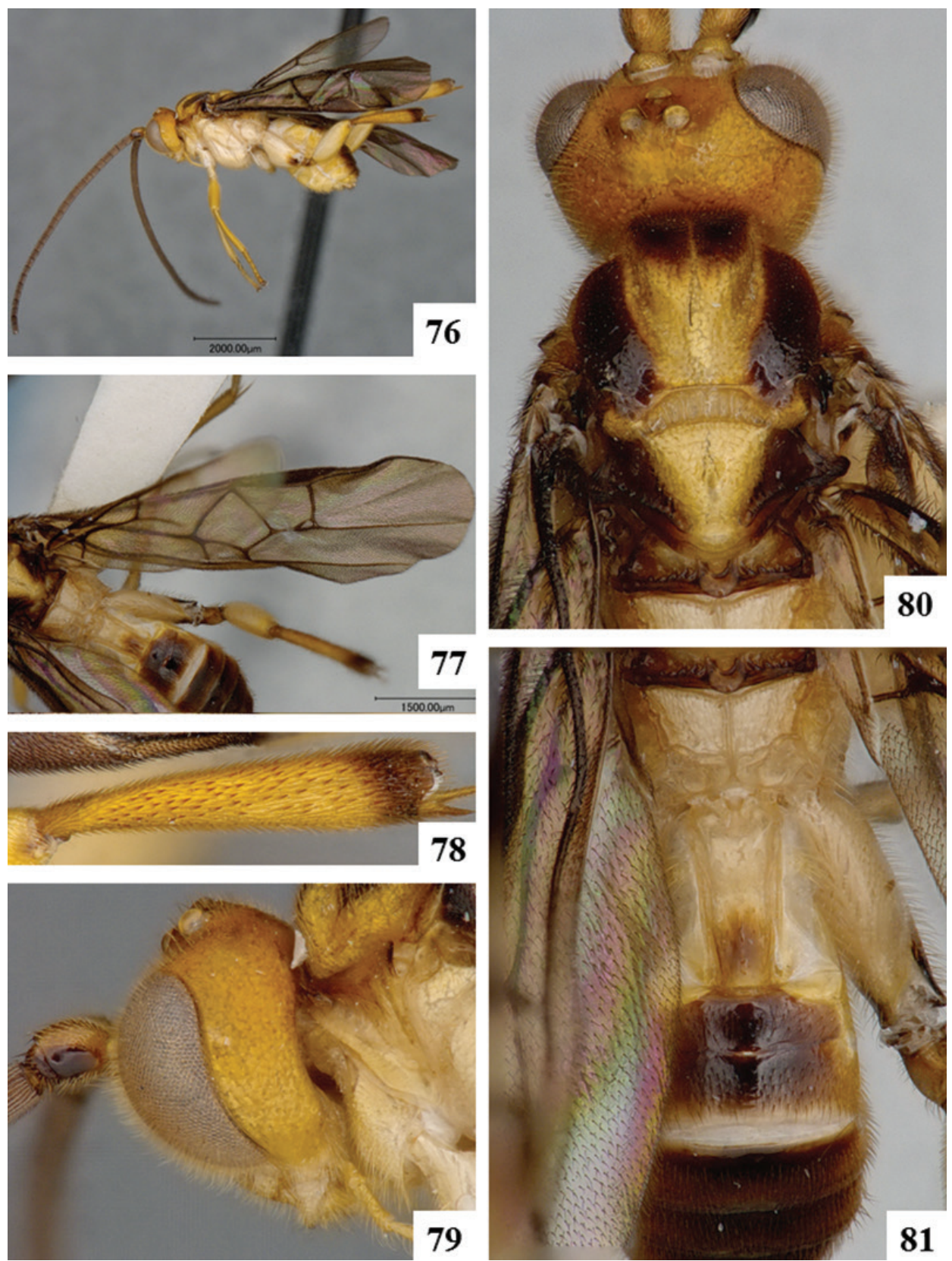

Figures 76-8 I. Prasmodon subfuscus. 76 Habitus 77 Fore wing 78 Metatibia 79 Head and mesosoma (partially), lateral view $\mathbf{8 0} \mathrm{Head}$ and mesosoma, dorsal view $\mathbf{8} \mathbf{I}$ Propodeum and metasoma, dorsal view.

from a single female. Both specimens are morphologically distinct, with different coloration of mesosoma, mediotergite 1, metacoxa, and trochanter (Figs 70, 73, 75 vs Figs $76,80,81$ ), as well as shape of mediotergite 2 . We have not found sexual di- 
morphism among any of the known species of Prasmodon (other than slight variations in the color of apical flagellomeres), and thus we do not think that the differences observed represent sexual dimorphism within a single species. Further evidence to consider those two specimens as separate species is provided by DNA barcoding; even though only short sequences (164 bp) could be recovered from both of them, they differed by 15 bp $(9.5 \%)$.

The exact collection locality of Prasmodon subfuscus is not clear from the original labels. "Silva Jardim" is a municipality of $938 \mathrm{~km}^{2}$ in the Brazilian state of Rio de Janeiro. Within the municipality is located the "Reserva Biologica de Poço das Antas", the largest lowland Atlantic Forest reserve in the state of Rio de Janeiro, containing $50 \mathrm{~km}^{2}$ of mostly secondary, evergreen forest. The area is very diverse (e.g., it contains at least 365 plant and 77 mammal species, Brito et al. 2004). This Atlantic Forest is one of the planet's biodiversity hotspots, with an extremely diverse and unique mix of forest types (http://www.conservation.org/where/priority_areas/ hotspots/Pages/hotspots_main.aspx). Thus, it is no surprise that two species of Prasmodon species occur in that region, just as multiple species do in the ACG rain forest in Costa Rica.

\section{Prasmodon tijucaensis Fernández-Triana \& Whitfield, sp. n. http://zoobank.org/0E2B527E-FEDB-478C-B739-08AE323E559D http://species-id.net/wiki/Prasmodon_tijucaensis}

Figs 82-87

Type locality. BRAZIL, Rio de Janeiro, Guanabara, Floresta da Tijuca (Tijuca Forest). Holotype. $q$ in CNC. Specimen labels: 1. BRAZIL, Guanabara, Floresta da Tijuca, ii.1974, M. Alvarenga. 2. DNA Voucher CNCHYM 01959.

Description. Female. Body length 3.3-3.4 mm. Fore wing length 3.7-3.8 mm. Body color: meso- and metasoma with brown to black areas, metasoma with most of tergites 2-8 dark brown (Fig 86, 87). Scape color: partially dark brown to black (Fig. 86). Flagellomeres color: all flagellomeres brown to black (Fig. 82). Tegula and humeral complex color: both dark. Mesotibia color: entirely yellow. Metatibia color: entirely yellow (Fig. 85). Metatibia spurs color: yellow-orange. Metatarsus color: entirely yellow-white. Fore wing color pattern: hyaline (Fig. 83). Fore wing veins color: all veins dark brown (Fig. 83). Pterostigma color: entirely dark brown. Hypostomal carina: not raised (Fig. 84). Scutoscutellar sulcus: with 7 impressions (Fig. 86). Areolet height $\div$ vein $r$ length (fore wing): $0.3 \times$. Hind wing subbasal cell: mostly with setae. Hind tarsal claws: with 1 or 2 teeth on anterior 0.3 of claw. Shape of mediotergite 1: distinctly narrowing posteriorly, width at posterior margin clearly less than width at anterior margin and median width (Fig. 87). Mediotergite 1 length $\div$ width at posterior margin 6.1-6.5 ×. Mediotergite 2 width at posterior margin $\div$ length: $2.1-2.5 \times$. Ovipositor sheaths length: $0.4 \times$ as long as metatibia.

Male. Unknown. 

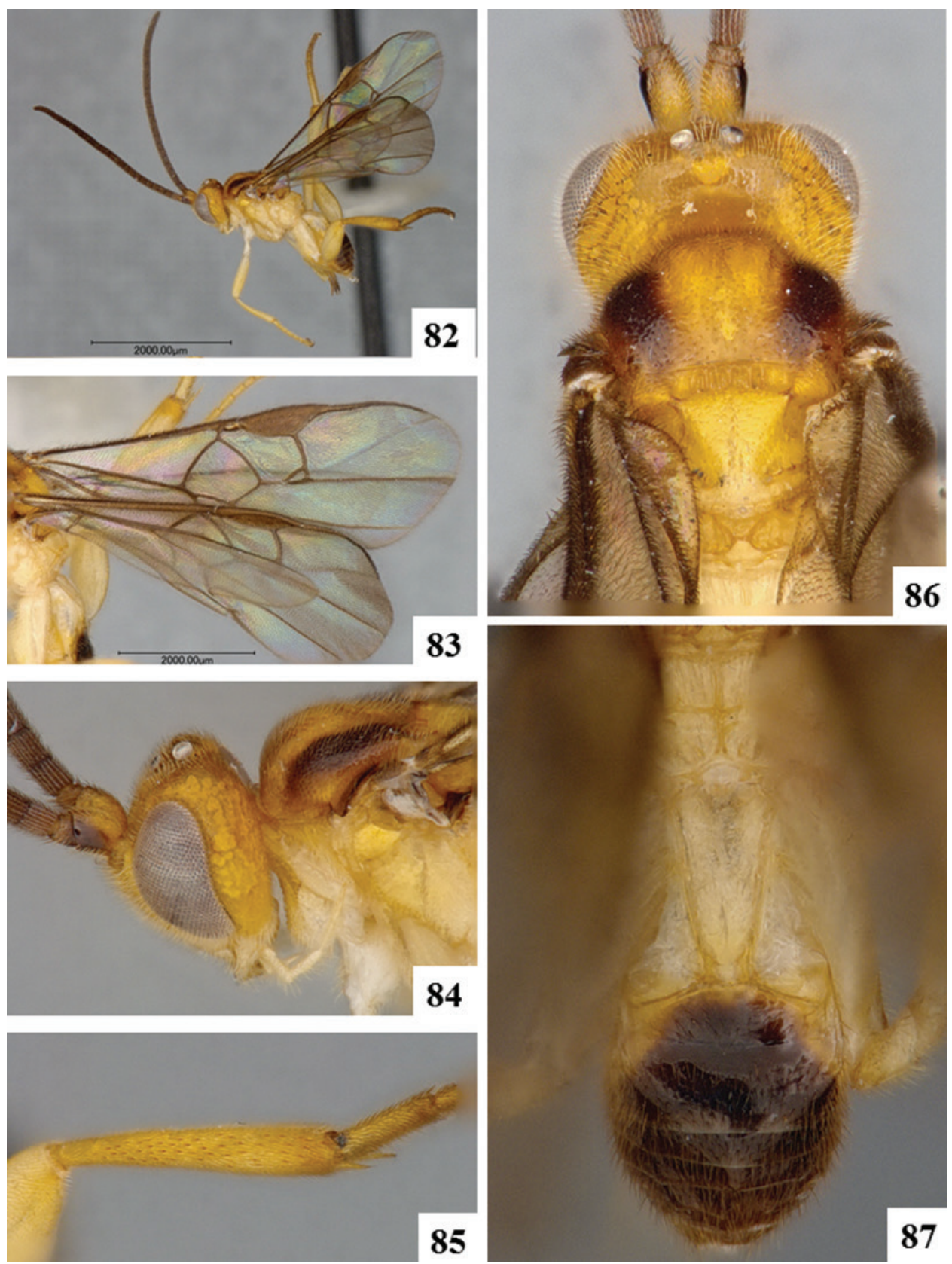

Figures 82-87. Prasmodon tijucaensis. 82 Habitus 83 Fore wing $\mathbf{8 4} \mathrm{Head}$ and mesosoma (partially), lateral view $\mathbf{8 5}$ Metatibia $\mathbf{8 6}$ Head and mesosoma, dorsal view $\mathbf{8 7}$ Propodeum and metasoma, dorsal view.

Molecular data. Sequences in BOLD: 1, barcode compliant sequences: 0 . Biology and ecology. Unknown.

Distribution. Brazil, Rio de Janeiro. 
Etymology. The name honours the type locality, Floresta da Tijuca, which is among the world's largest urban forests.

Comments. The only known specimen was considered by Mason (1981) to be a new species, which remained undescribed until now.

\section{Prasmodon verhoogdenokus Braet \& Fernández-Triana, sp. n. http://zoobank.org/7456681F-AAEC-4B62-ABC3-B1C9EB3387F2 http://species-id.net/wiki/Prasmodon_verhoogdenokus} Figs 88-93

Type locality. BRAZIL, Mato Grosso, Vila Vera, 500m, -12.7667, -55.5.

Holotype. + in CNC. Specimen labels: 1. BRAZIL, Vila Vera, M. Grosso, 500m, 1246'S, 55³0'W, X.1973, M. Alvarenga. 2. DNA Voucher, CNCHYM 01971.

Paratype. 6 \& (CNC, RMNH, IRSNB, MNHN). Ecuador, Napo, Tena, 400m, 16-17.ii.1987 (1 +); French Guiana, Saul, collecting dates: 2.ix.2010, 17.ix.2010, 26.x.2010 (3 क), Saul, Crique popote, Mont Belvedère, xii.2000 (1 q), Kourou, Piste Soumourou, 13.vi-20.vii.2002 (1 9 ).

Other material examined. $3 \widehat{\partial}$ from Colombia, Amazonas, PNN Amacayacu, San Martin, 150m (INHS); 2 from Ecuador, Napo, P. Misahualli, 350m (CNC); $98 \lesssim$ from French Guiana, Sinamary, Barrage de Petit-Saut; Inselberg Itoupé; RNR Trinité, Zone Aya; Nourages Reserve; Montagne de Kaw, Relais Patawa; and Saul (CNC and IRSNB); 2 ते from Peru, Madre de Dios, Rio Tampobata, Sachavacayoc center; and Loreto, Matse's Reserved Zone, site Jenaro Herrera, Rio Ucayali (CNC); 1 of from Suriname, Paramaribo Zoo (RMNH).

Description. Female. Body length 4.1-4.2 mm, 4.3-4.4 mm, 4.5-4.6 mm, rarely $3.9-4.0 \mathrm{~mm}$ or $4.7-4.8 \mathrm{~mm}$. Fore wing length $4.3-4.4 \mathrm{~mm}, 4.5-4.6 \mathrm{~mm}$, rarely $4.9-$ $5.0 \mathrm{~mm}$. Body color: meso- and metasoma entirely yellow-orange (with the exception of a very small black spot on axillar complex) (Figs 92, 93). Scape color: partially dark brown to black (Fig. 92). Flagellomeres color: with relatively extense yellow area (at least including F13-15, and usually apical half of F11 and basal half of F16) (Fig. 88, 91). Tegula and humeral complex color: tegula pale, humeral complex partially dark/ partialy pale. Mesotibia color: entirely yellow. Metatibia color: posterior $0.1-0.3$ dark brown to black. Metatibia spurs color: yellow-orange. Metatarsus color: dark brown to black (except for anterior 0.7 or less of first metatarsomere). Fore wing color pattern: mostly infumate, but with pale area centrally which gives the wing a banded appearance. Fore wing veins color: all veins dark brown (Fig. 89). Pterostigma color: entirely dark brown. Hypostomal carina: highly raised. Scutoscutellar sulcus: with 6 impressions, rarely with 5 impressions. Areolet height $\div$ vein $r$ length (fore wing): $0.15 \times$ or less. Hind wing subbasal cell: mostly without setae. Hind tarsal claws: with pectination (teeth) very irregular in spacing and length. Shape of mediotergite 1: distinctly narrowing posteriorly, width at posterior margin clearly less than width at anterior margin and median width (Fig. 93). Mediotergite 1 length $\div$ width at posterior margin 

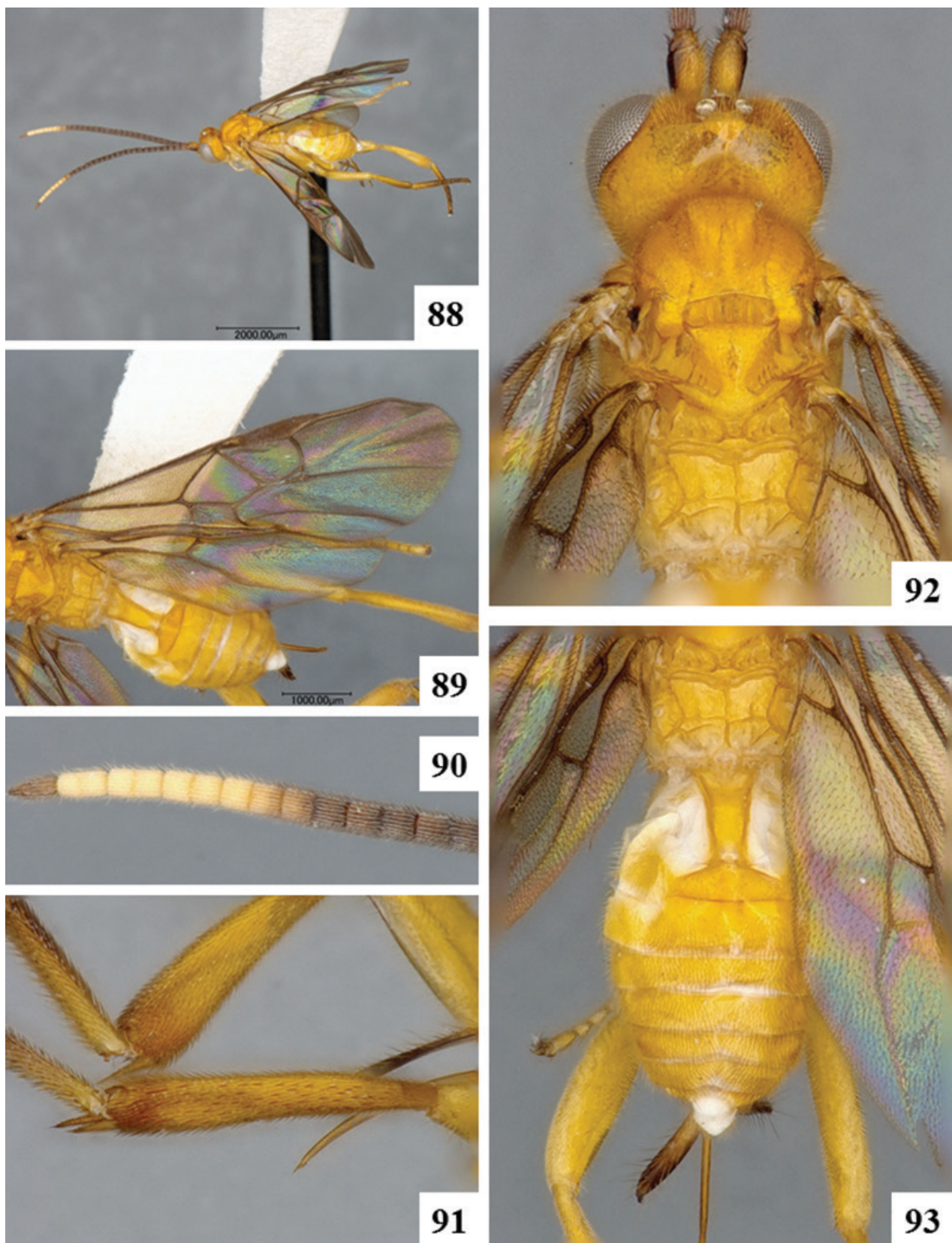

Figures 88-93. Prasmodon verhoogdenokus. 88 Habitus $\mathbf{8 9}$ Fore wing $\mathbf{9 0}$ Antenna (partially) 9 I Metatibia 92 Head and mesosoma, dorsal view 93 Propodeum and metasoma, dorsal view.

5.6-6.0 $\times$. Mediotergite 2 width at posterior margin $\div$ length: $2.6-3.0 \times$. Ovipositor sheaths length: $0.6 \times$ as long as metatibia, rarely $0.5 \times$ as long as metatibia.

Male. Morphologically similar to females, except for antenna. 
Molecular data. Sequences in BOLD: 1, barcode compliant sequences: 0. Two additional short sequences are available from two male specimens, but they were not included in the paratype series (see Comments below).

Biology and ecology. Light trapped and Malaise trapped.

Distribution. Brazil, Colombia, Ecuador, French Guiana, Peru, Suriname.

Etymology. From Dutch "verhoogde nok" = raised ridge, referring to the raised hypostomal carina.

Comments. The wide distribution of Prasmodon verhoogdenokus throughout South America, suggests it may represent a complex of morphologically cryptic species. At present there is no host known for the species, and the molecular data are meagre. Two male specimens from Ecuador that appear to be of this species rendered partial DNA sequences that differed by approximately $5 \%$ bp from the partial barcode of the female holotype (but the COI sequences for those three specimens only overlap for $240 \mathrm{bp}$ ). Although males included under this species look morphologically similar to the female holotype and the paratypes, they have been left out of the paratype series.

\section{Prasmodon zlotnicki Valerio \& Rodriguez, 2005}

http://species-id.net/wiki/Prasmodon_zlotnicki

Fig. 94, 102

Prasmodon zlotnicki Valerio \& Rodriguez, 2005: 31.

Type locality. COSTA RICA: ACG, Alajuela Province, Sector San Cristobal, Sendero Corredor, $620 \mathrm{~m}, 10.87868,-85.38963$. In the holotype description, the province was erroneously given as Guanacaste Province.

Holotype. + , INBio (examined).

Material examined. 8 ㅇ, $1 \curvearrowright$ (CNC, INBio, NMNH).

Description. Female. Body length $4.5-4.6 \mathrm{~mm}$. Fore wing length $5.1-5.2 \mathrm{~mm}$ or 5.3-5.4 mm. Body color: meso- and metasoma with brown to black areas, those on metasoma only thin brown areas centrally on tergites 4-8 (Fig. 94). Scape color: partially dark brown to black. Flagellomeres color: most flagellomeres brown to black, except for small apical area (F15-16, and occasionally apical half of F14) which is yellow-brown (Fig. 94). Tegula and humeral complex color: tegula pale, humeral complex partially dark/partialy pale. Mesotibia color: posterior $0.1-0.2$ dark brown to black. Metatibia color: posterior 0.1-0.3 dark brown to black (Fig. 94). Metatibia spurs color: dark brown to black (Fig. 94). Metatarsus color: dark brown to black (except for anterior 0.7 or less of first metatarsomere). Fore wing color pattern: uniformly and entirely infumate (except for small hyaline area near veins $(\mathrm{RS}+\mathrm{M}) \mathrm{b}$ and $2 \mathrm{M})$. Fore wing veins color: all veins dark brown. Pterostigma color: entirely dark brown. Hypostomal carina: highly raised. Scutoscutellar sulcus: with 6 impressions. Areolet height $\div$ vein $r$ length (fore wing): 0.2 $\times$. Hind wing subbasal cell: mostly without setae. Hind tarsal claws: with pectination (teeth) very irregular in spacing and length. Shape of mediotergite 1: distinctly narrow- 

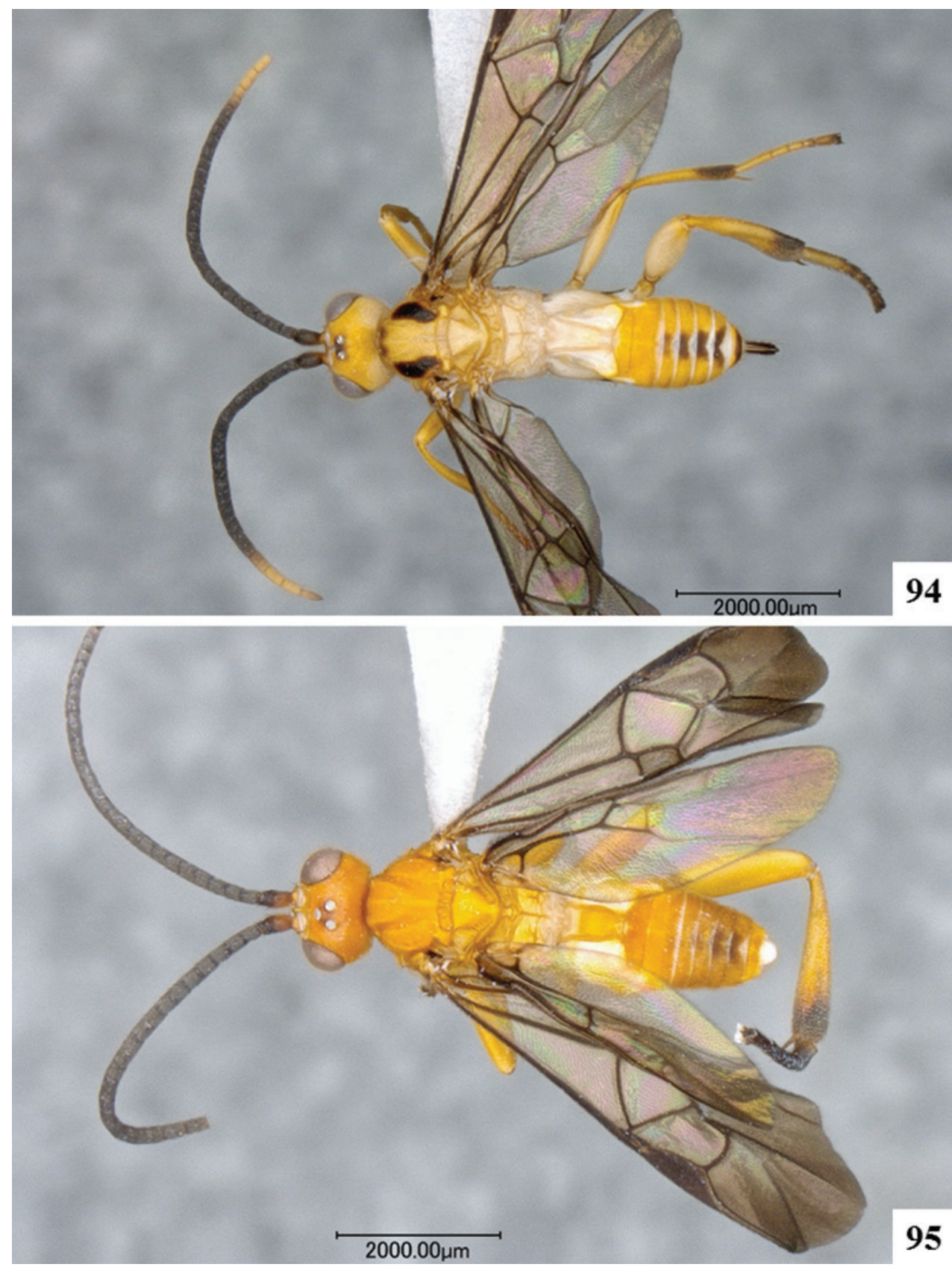

Figure 94-95. 94 Prasmodon zlotnicki. Body, dorsal view 95 Prasmodon eminens. Body, dorsal view. 

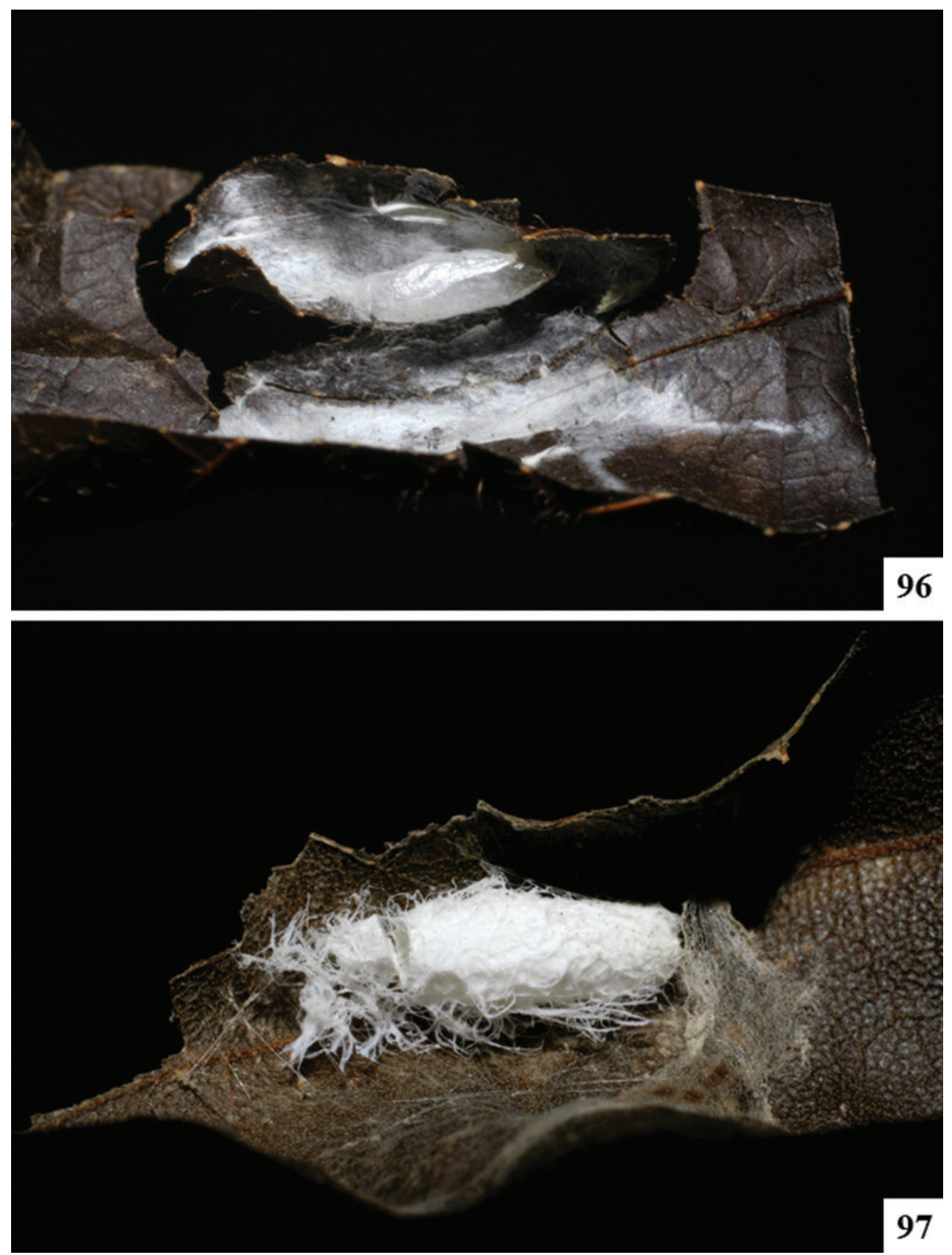

Figure 96-97. 96 Cocoon of Prasmodon mikepoguei 97 Cocoon of Prasmodon eminens. 

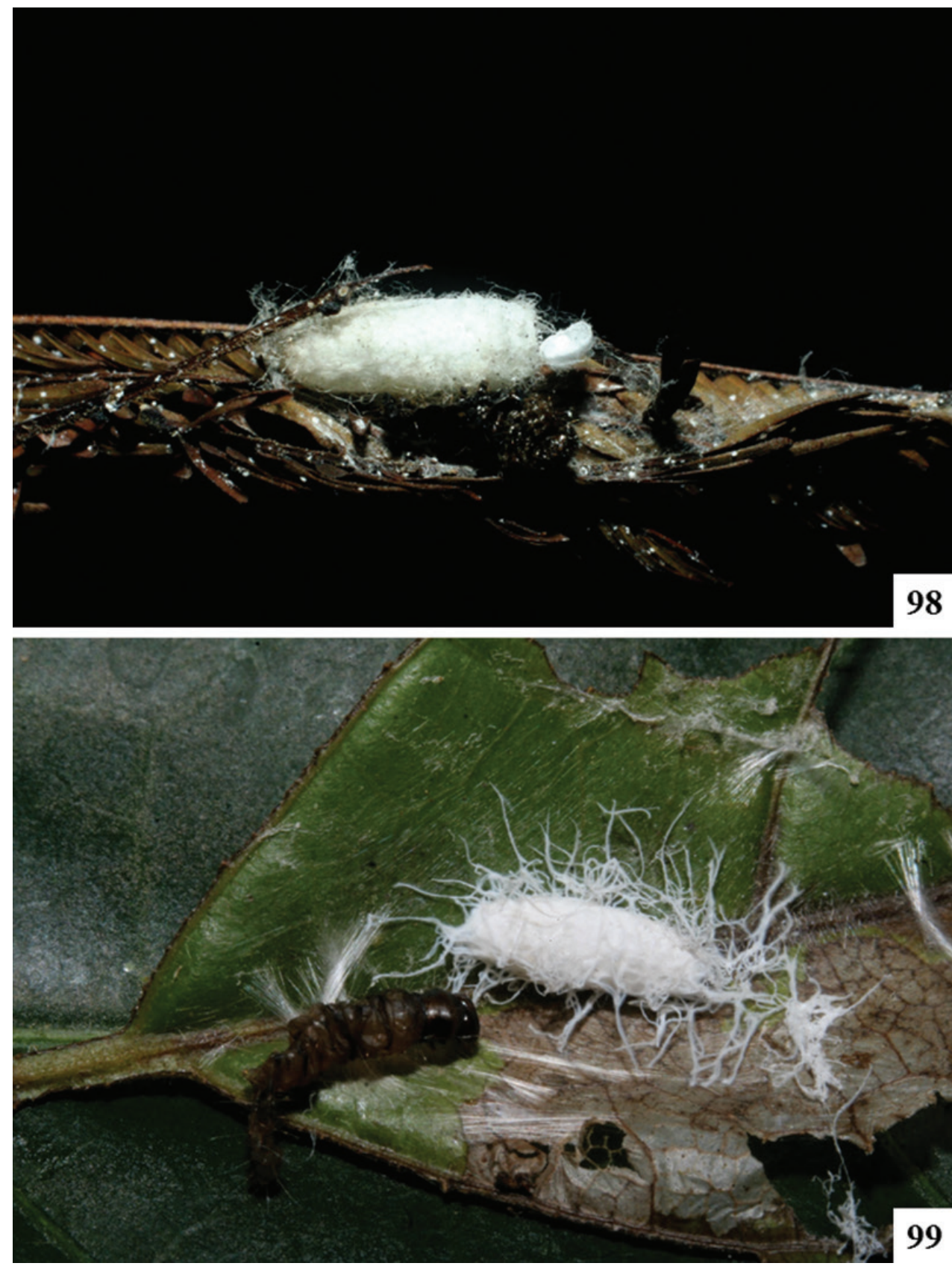

Figure 98-99.98 Cocoon of Prasmodon scottmilleri 99 Cocoon of Prasmodon bobpoolei. 


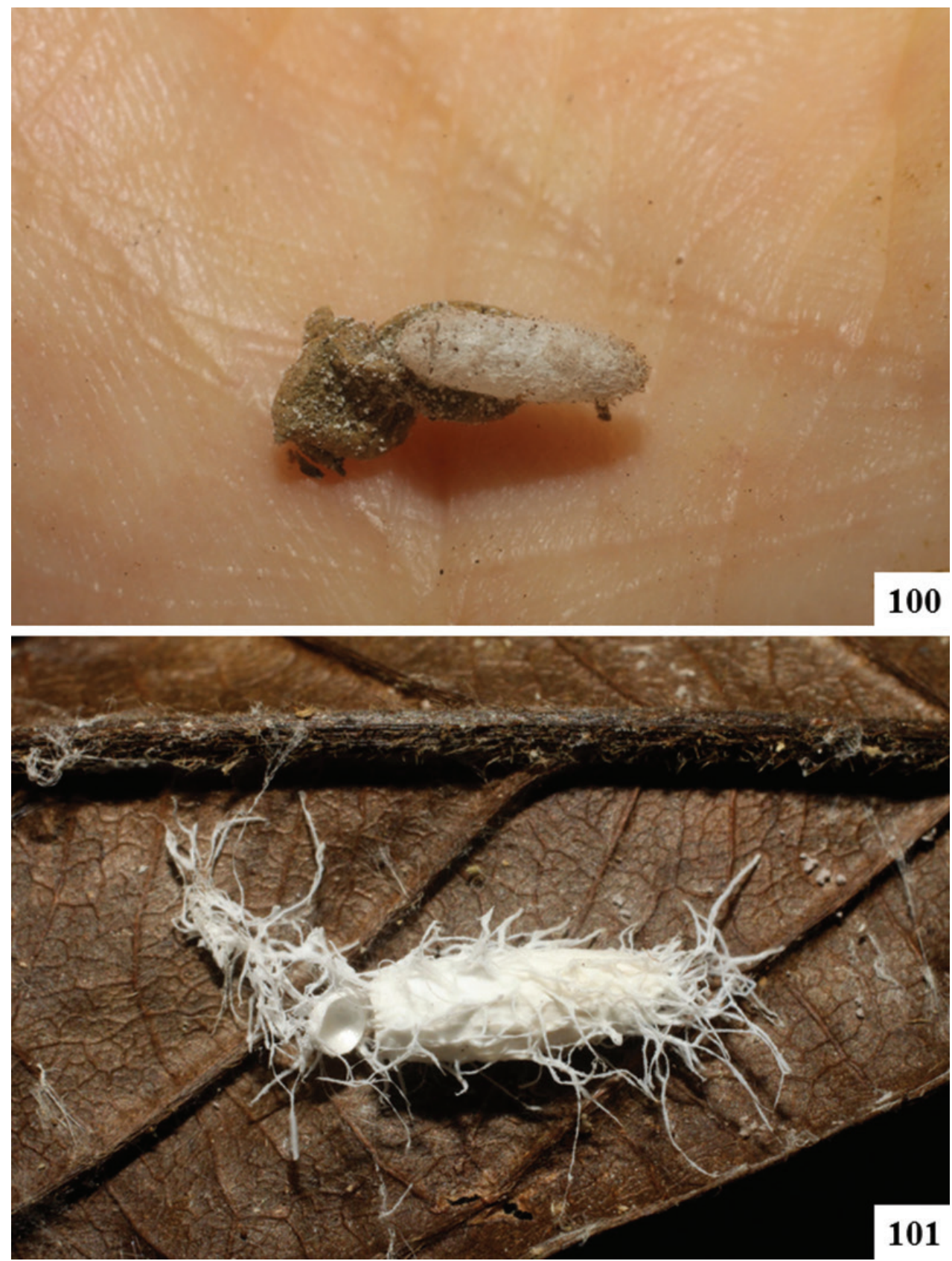

Figure 100-10I. 100 Cocoon of Prasmodon bobrobbinsi $\mathbf{I} \mathbf{1} \mathbf{I}$ Cocoon of Prasmodon almasolisae. 

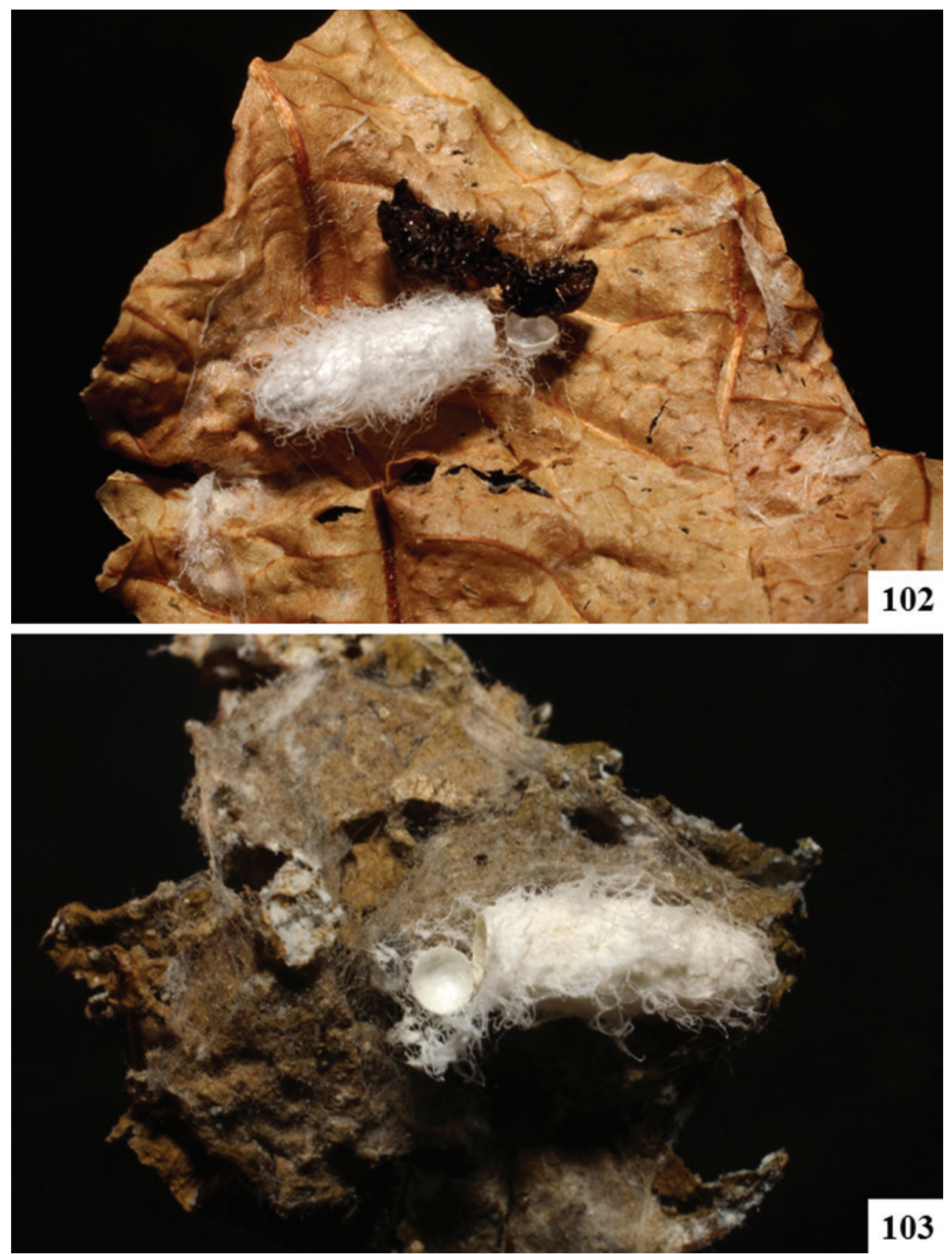

Figure 102-103. 102 Cocoon of Prasmodon zlotnicki 103 Cocoon of Prasmodon johnbrowni. 


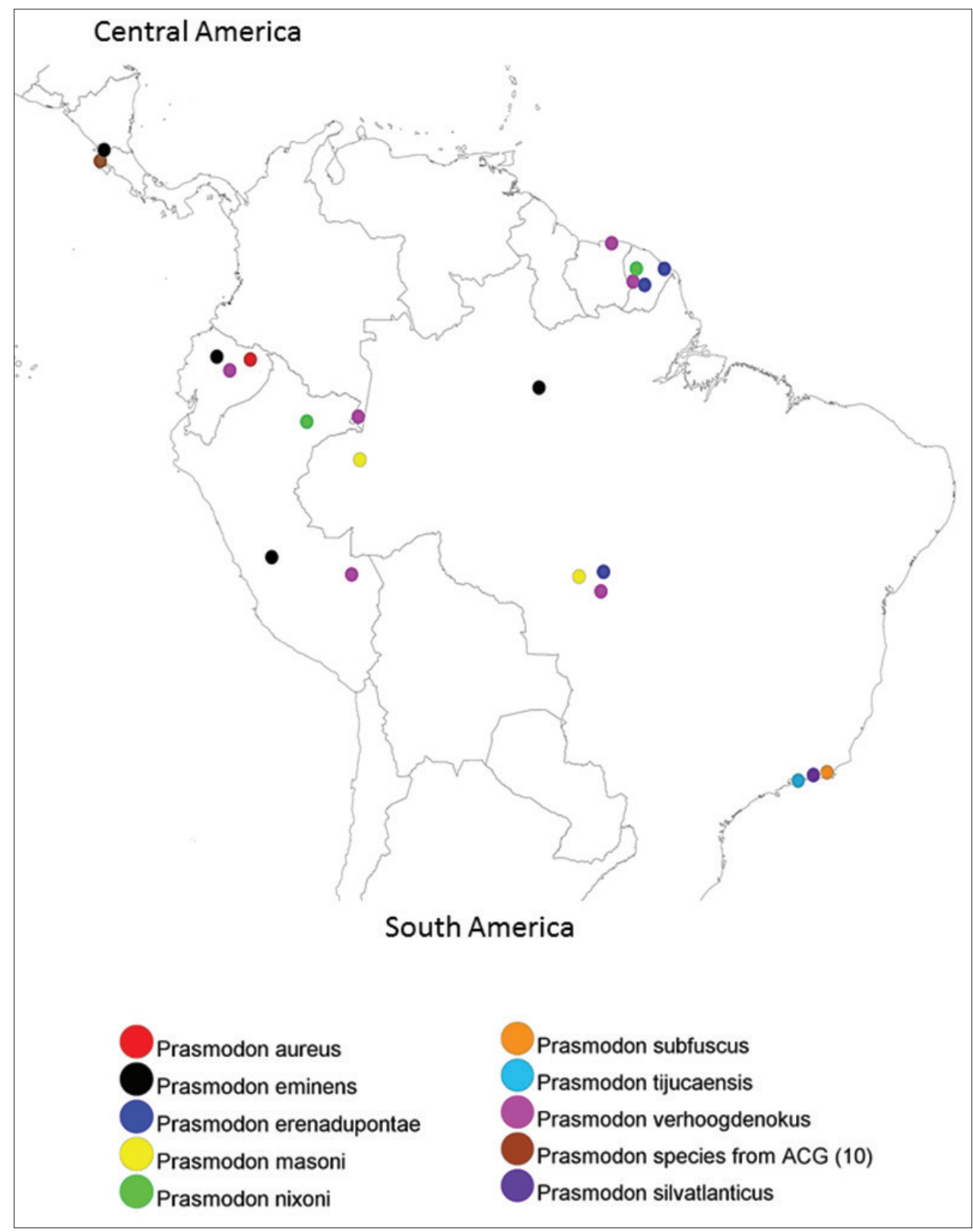

Figure 104. Distribution map of Prasmodon species in Central and South America. 


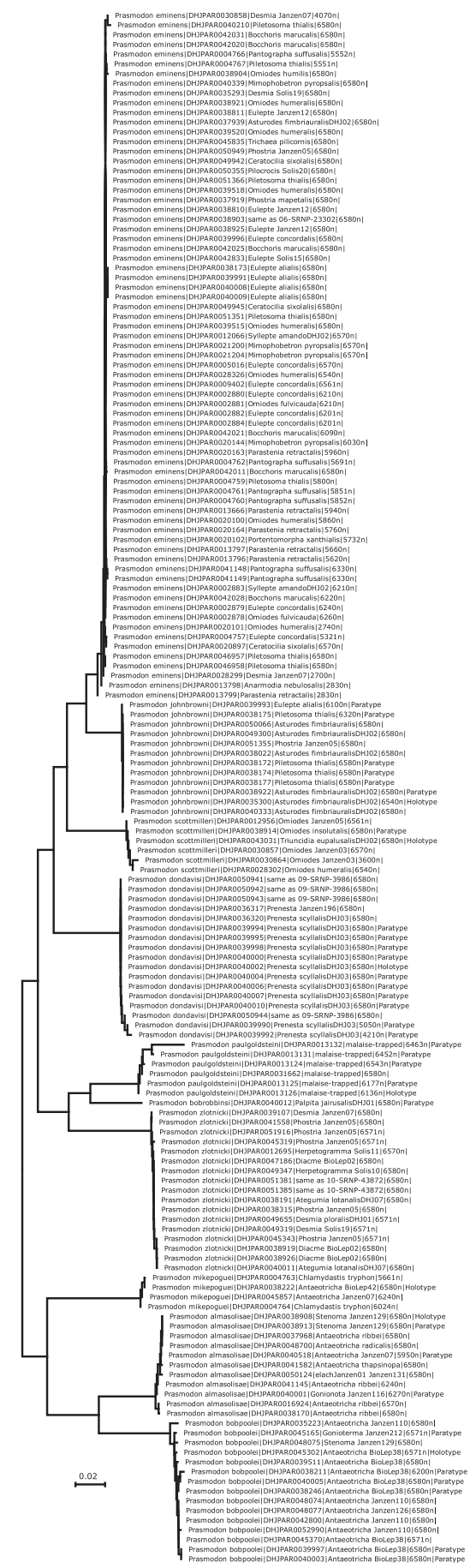

Figure 105. Neighbor-Joining tree (Saitou and Nei 1987) based on distances computed using the Kimura 2-parameter method (Kimura 1980) of all Prasmodon sequences from the ACG. Tree tips are labeled with species name|specimen accession|host information|sequence length and \# ambiguities|type status. 
ing posteriorly, width at posterior margin clearly less than width at anterior margin and median width (Fig. 94). Mediotergite 1 length $\div$ width at posterior margin 4.6-5.0 x, $5.1-5.5 x$, rarely $3.6-4.0 \times$. Mediotergite 2 width at posterior margin $\div$ length: $3.1-3.5 \times$ or 3.6-4.0 $\times$. Ovipositor sheaths length: $0.5 \times$ as long as metatibia.

Male. One male, the first found for the species. It is morphologically similar to females.

Molecular data. Sequences in BOLD: 11, barcode compliant sequences: 11 (Fig. 105).

Biology and ecology. Hosts: Crambidae, Ategumia lotanalis DHJ07, Desmia ploralisDHJ01, Desmia Janzen07, Desmia Solis19 Diacme biolep02, Herpetogramma Solis 10, Herpetogramma Solis 11, Phostria Janzen05.

Distribution. Costa Rica, ACG rain forest (Valerio et al. 2005).

\section{Acknowledgements}

JFT is greatly indebted to John Huber and Caroline Boudreault (CNC) for helping with the preparation of many specimens used in this study. Thanks also go to Odette Morvan and Jean Cerda for their help for their long term collections in the "Montagne de Kaw", the members of the "Société Entomologique Antilles Guyane" for their support in logistic and their collecting in several stations in French Guiana, and all other people who have kindly housed or supported the work of YB. The study has been supported by U.S. National Science Foundation grants BSR 9024770 and DEB 9306296, 9400829, 9705072, 0072730, 0515699 to DHJ, and grants from the Wege Foundation, International Conservation Fund of Canada, Jessie B. Cox Charitable Trust, Blue Moon Fund, Guanacaste Dry Forest Conservation Fund (GDFCF), Area de Conservación Guanacaste, Permian Global, and University of Pennsylvania (DHJ), and by the intense efforts of the ACG/ GDFCF parataxonomist team that found and reared the parasitoids described here, to say nothing of the international taxasphere that labors to obtain and develop the caterpillar and parasitoid taxonomy essential to this effort in science and conservation. The reviews of Gavin Broad and two anonymous reviewers greatly improved the manuscript.

\section{References}

Austin AD (1990) Revision of the enigmatic Australasian genus Miropotes Nixon (Hymenoptera: Braconidae: Microgastrinae), with comments on the phylogenetic importance of the female ovipositor system. Systematic Entomology 15: 43-68. doi: 10.1111/j.13653113.1990.tb00302.x

Banks JC, Whitfield JB (2006) Dissecting the ancient rapid radiation of microgastrine wasp genera using additional nuclear genes. Molecular Phylogenetics and Evolution 41: 690-703. doi: 10.1016/j.ympev.2006.06.001

Brito D, Oliveira LC, Mello MAR (2004) An overview of mammalian conservation at Poço das Antas Biological Reserve, southeastern Brazil. Journal for Nature Conservation 12(4): 219-228. doi: 10.1016/j.jnc.2004.09.001 
Deans AR, Whitfield JB, Janzen DH (2003) Taxonomy and natural history of the Microgastrinae genus Alphomelon Mason (Hymenoptera: Braconidae). Journal of Hymenoptera Research 12(1): 1-41.

Fernández-Triana J, Cardinal S, Whitfield JB, Hallwachs W, Smith MA, Janzen DH (2013) A review of the New World species of the parasitoid wasp Iconella (Hymenoptera: Braconidae, Microgastrinae). ZooKeys 321: 65-87. doi: 10.3897/zookeys.321.5160

Fernández-Triana J, Whitfield JB, Rodriguez JJ, Smith MA, Janzen DH, Hallwachs W, Hajibabaei M, Burns JM, Solis MA, Brown J, Cardinal S, Goulet H, Hebert PDN (2014) Review of Apanteles (Hymenoptera: Braconidae, Microgastrinae) from Area de Conservación Guanacaste, northwestern Costa Rica, with keys to all described species from Mesoamerica. ZooKeys 383: 1-565. doi: 10.3897/zookeys.383.6418

Grinter CC, Whitfield JB, Connahs H, Dyer LA, Hallwachs W, Janzen DH (2009) A key to New World Distatrix Mason (Hymenoptera: Braconidae), with descriptions of six new reared Neotropical species. Journal of Insect Science (Tucson) 9(29): 1-17. doi: 10.1673/031.009.2901

Huber JT, Sharkey MJ (1993) Structure. In: Goulet H, Huber JT (Eds) Hymenoptera of the world: an identification guide to families. Agriculture Canada Research Branch, Monograph No. 1894E, Ottawa, Canada, 13-59.

Ivanova NV, deWaard JR, Hebert PDN (2006) An inexpensive, automation-friendly protocol for recovering high-quality DNA. Molecular Ecology Notes 6: 998-1002. doi: 10.1111/j.1471-8286.2006.01428.x

Janzen DH, Walker AK, Whitfield JB, Delvare G, Gauld ID (2003) Host specificity and hyperparasitoids of three new Costa Rican species of Microplitis Foerster (Hymenoptera: Braconidae: Microgastrinae), parasitoids of sphingid caterpillars. Journal of Hymenoptera Research 12(1): 42-76.

Janzen DH, Hallwachs W, Blandin P, Burns JM, Cadiou J, Chacon I, Dapkey T, Deans AR, Epstein ME, Espinoza B, Franclemont JG, Haber WA, Hajibabaei M, Hall JPW, Hebert PDN, Gauld ID, Harvey DJ, Hausmann A, Kitching I, Lafontaine D, Landry J, Lemaire C, Miller JY, Miller JS, Miller L, Miller SE, Montero J, Munroe E, Rab Green S, Ratnasingham S, Rawlins JE, Robbins RK, Rodriguez JJ, Rougerie R, Sharkey MJ, Smith MA, Solis MA, Sullivan JB, Thiaucourt P, Wahl DB, Weller SJ, Whitfield JB, Willmott KR, Wood DM, Woodley NE, Wilson JJ (2009) Integration of DNA barcoding into an ongoing inventory of complex tropical biodiversity. Molecular Ecology Resources 9 (Supplement 1): 1-26. doi: 10.1111/j.1755-0998.2009.02628.x

Janzen DH, HallwachsW (2011) Joining inventory by parataxonomists with DNA barcoding of a large complex tropical conserved wildland in northwestern Costa Rica. PLoS ONE 6(8): e18123. doi: 10.1371/journal.pone.0018123

Karlsson D, Ronquist F (2012) Skeletal morphology of Opius dissitus and Biosteres carbonarius (Hymenoptera: Braconidae), with a discussion of terminology. PLoS ONE 7(4): e32573. doi: 10.1371/journal.pone.0032573

Kimura M (1980). A simple method for estimating evolutionary rate of base substitutions through comparative studies of nucleotide sequences. Journal of Molecular Evolution 16: 111-120. doi: 10.1007/BF01731581 
Mardulyn P, Whitfield JB (1999) Phylogenetic signal in the COI, 16S and 28S genes for inferring relationships among genera of Microgastrinae (Hymenoptera: Braconidae); evidence of a high diversification rate in this group of parasitoids. Molecular Phylogenetics and Evolution 12: 282-294. doi: 10.1006/mpev.1999.0618

Mason WRM (1981) The polyphyletic nature of Apanteles Foerster (Hymenoptera: Braconidae): A phylogeny and reclassification of Microgastrinae. Memoirs of the Entomological Society of Canada, Ottawa, Canada, 147 pp.

Nixon G (1965) A reclassification of the tribe Microgasterini (Hymenoptera: Braconidae). Bulletin of the British Museum (Natural History), Entomology series, Supplement 2: 1-284.

Ratnasingham S, Hebert PDN (2007) BOLD: The Barcode of Life Data System (www. barcodinglife.org). Molecular Ecology Notes 7: 355-364. doi: 10.1111/j.14718286.2007.01678.x

Rodriguez JJ, Fernández-Triana J, Smith MA, Janzen DH, Hallwachs W, Erwin TL, Whitfield JB (2013) Extrapolations from field studies and known faunas converge on dramatically increased estimates of global microgastrine parasitoid wasp species richness (Hymenoptera: Braconidae). Insect Conservation and Diversity 6: 530-536. doi: 10.1111/icad.12003

Shorthouse DP (2010) SimpleMappr, an online tool to produce publication-quality point maps. http://www.simplemappr.net [accessed 3 December, 2013]

Saitou N, Nei M (1987). The neighbor-joining method: A new method for reconstructing phylogenetic trees. Molecular Biology and Evolution 4: 406-425.

Smith MA, Woodley NE, Janzen DH, Hallwachs W, Hebert PDN (2006) DNA barcodes reveal cryptic host-specificity within the presumed polyphagous members of a genus of parasitoid flies (Diptera: Tachinidae). Proceedings of the National Academy of Sciences 103: 3657-3662. doi: 10.1073/pnas.0511318103

Smith MA, Wood DM, Janzen DH, Hallwachs W, Hebert PDN (2007) DNA barcodes affirm that 16 species of apparently generalist tropical parasitoid flies (Diptera, Tachinidae) are not all generalists. Proceedings of the National Academy of Sciences 104: 4967-4972. doi: 10.1073/pnas.0700050104

Smith MA, Rodriguez JJ, Whitfield JB, Deans AR, Janzen DH, Hallwachs W, Hebert PDN (2008) Extreme diversity of tropical parasitoid wasps exposed by iterative integration of natural history, DNA barcoding, morphology, and collections. Proceedings of the National Academy of Sciences 105: 12359-12364. doi: 10.1073/pnas.0805319105

Smith MA, Fernández-Triana JL, Eveleigh E, Gómez J, Guclu C, Hallwachs W, Hebert PDN, Hrcek J, Huber JT, Janzen DH, Mason PG, Miller SE, Quicke DLJ, Rodriguez JJ, Rougerie R, Shaw MR, Varkonyi G, Ward D, Whitfield JB, Zaldívar-Riverón A (2013) DNA barcoding and the taxonomy of Microgastrinae wasps (Hymenoptera, Braconidae): impacts after 8 years and nearly 20000 sequences. Molecular Ecology Resources 13: 168-176. doi: 10.1111/1755-0998.12038

Valerio AA, Rodriguez JJ, Whitfield JB, Janzen DH (2005) Prasmodon zlotnicki, a new Neotropical species of the genus Prasmodon Nixon (Braconidae: Microgastrinae) from Costa Rica, with the first host records for the genus. Zootaxa 1016: 29-38.

Valerio AA, Whitfield JB, Janzen DH (2009) Review of world Parapanteles Ashmead (Hymenoptera: Braconidae: Microgastrinae), with description of fourteen new Neotropical species and the first description of the final instar larvae. Zootaxa 2084: 1-49. 
Whitfield JB (1995) Checklist of the Microgastrinae (Hymenoptera: Braconidae) in America north of Mexico. Journal of the Kansas Entomological Society 68: 245-262.

Whitfield JB (1997) Subfamily Microgastrinae. In: Wharton RA, Marsh PM, Sharkey MJ (Eds) Manual of the New World genera of the family Braconidae (Hymenoptera). Special Publication No. 1, International Society of Hymenopterists, Washington, D.C., 333-364.

Whitfield JB, Mardulyn P, Austin AD, Dowton M (2002) Phylogenetic relationships among microgastrine braconid wasp genera based on data from the 16S, COI and $28 \mathrm{~S}$ genes and morphology. Systematic Entomology 27: 337-359. doi: 10.1046/j.13653113.2002.00183.x

Whitfield JB, Fernández-Triana J, Janzen DH, Hallwachs W, Smith A, Cardinal S (2012) Mariapanteles (Hymenoptera, Braconidae), a new genus of Neotropical microgastrine parasitoid wasp discovered through biodiversity inventory. ZooKeys 208: 61-80. doi: $10.3897 /$ zookeys.208.3326

\section{Supplementary material I}

Supplementary information for studied specimens of Prasmodon (Hymenoptera: Braconidae, Microgastrinae) from ACG, Costa Rica

Authors: Jose L. Fernandez-Triana, James B. Whitfield, Alex M. Smith, Winnie Hallwachs, Daniel H. Janzen

Data type: Collection details, taxonomic information, DNA sequence, accession numbers

Copyright notice: This dataset is made available under the Open Database License (http://opendatacommons.org/licenses/odbl/1.0/). The Open Database License $(\mathrm{ODbL})$ is a license agreement intended to allow users to freely share, modify, and use this Dataset while maintaining this same freedom for others, provided that the original source and author(s) are credited.

Link: doi: 10.3897/JHR.37.6784.app1 